\title{
Hate Contagion: Measuring the spread and trajectory of hate on social media
}

\author{
John D. Gallacher* \\ Oxford Internet Institute \\ University of Oxford
}

\author{
Jonathan Bright \\ Oxford Internet Institute \\ University of Oxford
}

\begin{abstract}
Online hate speech is a growing concern, with minorities and vulnerable groups increasingly targeted with extreme denigration and hostility. The drivers of hate speech expression on social media are unclear, however. This study explores how hate speech develops on a fringe social media platform popular with the far-right, Gab. We investigate whether users seek out this platform in order to express hate, or whether instead they develop these opinions over time through a mechanism of socialisation, as they interact with other users on the platform. We find a positive association between the time users spend on the platform and their hate speech expression. We show that while some users do arrive on these platforms with pre-existing hate stances, others develop these expressions as they get exposed to the hateful opinions of others. Our analysis reveals how hate speech develops online, the important role of the group environment in accelerating its development, and gives valuable insight to inform the development of counter measures.
\end{abstract}

Keywords: Hate Speech | Social Media | Social Networks | Extremism | Radicalisation | Intergroup Conflict

16 February 2021

* Corresponding Author: John Gallacher - john.gallacher@oii.ox.ac.uk 


\section{Introduction}

Far-right extremist violence has been increasing across the western world in recent years, with a $320 \%$ increase since 2015 (Global Terrorism Index, 2019). This phenomenon has occurred in parallel with an increase in online hate speech (Williams, 2019). A growing number of studies show how those with prejudiced or negative views towards minority groups are using the social web to spread antagonistic, inflammatory and hateful messages targeting these groups (e.g. Alorainy, Burnap, Liu, \& Williams, 2018; Zannettou, ElSherief, Belding, Nilizadeh, \& Stringhini, 2020). This could lead to a reduction in group relations and an increase in intergroup conflict, with particular targeting of vulnerable and minority groups.

Social media has also begun to play a larger role in encouraging offline hate crimes (Williams \& Burnap, 2019; Gaudette, Scrivens, \& Venkatesh, 2020). Suspects in several recent hate-related terror attacks had an extensive history of social media activity within hate groups, including notably the Tree of life Synagogue shooting Pittsburgh and the Charlottesville 'Unite the Right' rally, both in the United States (Evans, 2019). Social media was also used to livestream the 2019 terror attack at two mosques in Christchurch, New Zealand. This latter event sparked a number of copy-cat attempts, demonstrating the power and influence of online hate (MacAvaney et al., 2019). However, the exact role that social media activity played in facilitating or influencing these hateful beliefs is unclear.

Online hate typically takes the form of verbal or written attacks against a specific group of people focused on, or motivated by, certain aspects of that group's identity (Davidson, Warmsley, Macy, \& Weber, 2017; de Gibert, Perez, García-Pablos, \& Cuadros, 2018; Gallacher, 2021). Hate speech is particularly common in expressions shared by the far-right, both within traditional right-wing extremist groups and the more recent 'alt-right' movements (Mathew, Dutt, Goyal, \& Mukherjee, 2019; Vidgen, Yasseri, \& Margetts, 2019). These far-right groups are broadly defined by holding ideals of racial and ethnic nationalism, and typically framed in terms of white power and/or white identity; this forms the ingroup. Outgroups are framed in terms of perceived threats to this identity posed by a combination of non-whites, Jews, Muslims, immigrants, refugees, members of the LGBTQ community, and feminists, among others (Conway, Scrivens, \& Macnair, 2019; Scrivens, 2020). The Internet is a key breeding ground for these hateful and extremists' narratives (All-Party Parliamentary Group (APPG) on Hate Crime, 2019).

Despite its prevalence, the influence that interacting with online hate speech has on processes of extremism and radicalisation are contested (Meleagrou-Hitchens \& Kaderbhai, 2016), and the use of empirical evidence to investigate how online hate spreads is limited. Evidence suggests that exposure to extreme material on social media can exert influence on users, including the promotion of outgroup derogation, but how this relates to users' own opinions and long-term behaviours is unclear.

Understanding how hate spreads on social media platforms is paramount to developing any mitigation strategies. In particular, how the hate speech expressions of individual users fluctuates over time, and this influences those they are connected to, is poorly understood (Kleinberg, Vegt, \& Gill, 2020).

In addition, not all users active on fringe social media platforms express hate against outgroups (Kleinberg et al., 2020). One reason for this variation between users may be due to differences in exposure to hate speech on the platform, with this influencing their own hate expression (Ferrara, 2017; Ferrara, Wang, Varol, Flammini, \& Galstyan, 2016), with some users experiencing greater social pressure to conform, i.e. to produce hate speech themselves. This has not been tested, however. 
In the current study we investigate the nature of hate speech expression on the fringe social media platform Gab and examine the role that social influence plays in propagating hate. We first test whether users express hate speech and display pre-existing prejudices from the moment they join the platform, or instead whether hate speech expression grows with time spent on the platform. We subsequently look at the social contagion effects of online hate speech, and whether greater exposure to online hate increases a user's likelihood of adopting this behaviour and producing hate speech themselves. Finally, we examine whether hate exposure can lead to transitive effects across target groups - i.e. whether exposure to hate against a particular group can lead to wider hate against other groups. By measuring the role of this social influence, we capture the way in which social media users affect each other's beliefs, emotions, attitudes, and behaviours, and as such give insight into the complex ways that hateful behaviours develop. Studying these effects is key to understanding the spread of hate speech online and its consequences, which is vital to inform countermeasures.

\section{Literature Review}

In order to understand what drives users to express hate speech on social media, it is first essential to understand the social psychological underpinnings of outgroup denigration and how collective hate can develop, and the existing evidence for the role of social media in this process.

\section{Hate as a group process}

Recent evidence suggests that there is no pathological 'terrorist' or 'extremist' personality, but rather terrorism and hateful extremist violence occur as group phenomena (Doosje et al., 2016). Ingroup and outgroup distinctions can be a strong driver within the radicalisation process, especially when the outgroup is felt to present a direct threat (Sageman, 2004). Therefore, it is important to study hate speech in the context of intergroup relations and to understand the collective group dynamics to which individuals belong and take their social identity from. This social identity gives individuals a shared and collective representation of who they are, how they should behave, and what values they hold, including which outgroups they denigrate (Hogg, Abrams, \& Brewer, 2017; Tajfel, 1974). The importance of this group identity is also the exhibited by the victims of hate, who are typically targeted because of the groups they belong to rather than their individual actions or characteristics (Reicher, Haslam, \& Rath, 2008).

When viewing offline hate as a group process, evidence suggests that counter to the traditional literature (Haney, Banks, \& Zimbardo, 1973; Milgram, 1963), groups do not commit acts of extreme inhumanity due to a lack of awareness or control, but rather because they believe what they are doing is right, morally correct, and to be celebrated (Reicher et al., 2008). In this process, the ingroup is championed as (uniquely) good and the eradication of the outgroup is embraced as necessary to defend ingroup virtue. Extremist positions are adopted when there is the belief that the survival of the ingroup is inseparable from direct or offensive action against the outgroup (Berger, 2018), and radicalisation is therefore the increased preparation and commitment to intergroup conflict (McCauley \& Moskalenko, 2008). Group socialisation is thought to be especially important in the shift towards extremism, with new group members learning the norms of the group and developing shared expectations and belief system about how to behave (Levine \& Moreland, 1994; Moreland \& Levine, 1982). If the norms of the ingroup include derogation towards outgroups, then these can also be learned through socialisation (Bracegirdle, 2020). Smith, Blackwood \& Thomas (2019) propose a group socialisation process of radicalisation where group members gradually adopt shared identification with a set of radical norms, including outgroup hate, through social interactions with the group that leverage their shared perceptions, experiences, and grievances, whilst also changing the nature of the group as a whole towards an extreme position. 
Hate speech has two distinct roles in this group radicalisation process. Firstly, it signals ingroup membership to other ingroup members through a shared outgroup 'enemy'. Secondly, it dehumanizes and diminishes members of the targeted outgroup, expresses the threat posed by this group, highlights the shared grievance with other members of the ingroup, and calls for direct action against the perceived threat (Gagliardone, Gal, Alves, \& Martinez, 2015). Hate speech therefore does not only represent a reduction in intergroup relations but also leads such relations to reduce further. Frequent and repetitive exposure to hate speech also increases outgroup prejudice by leading to desensitization to violence and subsequently to lower evaluations of the victims of this violence (Soral, Bilewicz, \& Winiewski, 2018). Because social interactions focused around shared grievances are particularly powerful in driving extreme forms of social action (Thomas, Mcgarty, \& Louis, 2014), understanding the spread of hate speech on social media and how it influences those exposed to it is therefore crucial.

\section{The adoption of outgroup hate through social contagion}

Offline, this spread of outgroup hate from one ingroup member to another can be thought of as social contagion, a form of social influence whereby individuals adopt novel behaviours as a result of interacting with others who are already behaving in this way (Rogers, 2003; Valente, 1995). In other contexts, the influence of this exposure on adoption of the behaviour increases with social pressure to conform (Asch, 1955). In this way, it is not the individuals themselves, but rather the relationships between individuals and their interpersonal interactions, which determine whether someone adopts the behaviour or not. Historically, this effect has been shown to occur in the adoption of novel farming practices (Ryan \& Gross, 1943), medical innovations (Coleman, Katz, \& Menzel, 1966) and the use of communication networks (Rogers \& Kincaid, 1984). More recently, this social contagion effect has been shown offline in the case of outgroup denigration, whereby social consensus plays a large role in shaping local social norms and acceptability of certain prejudices (Crandall \& Stangor, 2008). Social contagion via networks of relationships can occur through direct ties and immediate contact with others, but also through indirect ties - connections of those we ourselves are connected with (Granovetter, 1973). This latter form of contact can expose individuals to wider information and greater novelty than direct connections.

More recently, the concept of social contagion has been applied to the study of online behaviours. In particular, it has been used to study the spread of information through online social networks (Lazer et al., 2009), with applications ranging from politics (Bond et al., 2012), technology (Onnela \& ReedTsochas, 2010), public health (Centola, 2010), and moralised discourse (Brady, Crockett, \& Van Bavel, 2020). Individuals who experience higher levels of information exposure are more likely to pass it on (Bakshy, Rosenn, Marlow, \& Adamic, 2012), and weak ties are responsible for greater propagation of novel information through the network than strong ties (Bakshy et al, 2012). There is a strong social component to this process: users are more likely to share a piece of information if they believe a greater number of their close contacts have already shared it (Hodas \& Lerman, 2014). In addition to informational influence, emotional states can also be transferred via contact with others. Those exposed to greater positive/negative emotional content have been shown to express this emotion more themselves (Kramer, Guillory, \& Hancock, 2014), while aggressive states on Twitter have been shown to propagate from one user to another (Terizi, Chatzakou, Pitoura, Tsaparas, \& Kourtellis, 2020).

In the context of extremist content, these online social contagion effects have been shown both for Islamic extremist and far-right material (Ferrara, 2017; Mathew et al., 2019). By mapping the spread of propaganda, influential Islamic extremist supporters on Twitter were shown to influence other previously non-supportive users, with the average Islamic extremist "infecting" 2.13 other users before 
being suspended (Ferrara, 2017). On far-right platforms, users expressing hate speech were shown to instigate larger information cascades than non-hateful users, indicating that these users are more likely to 'go viral' on the platform (Mathew et al., 2019). However, in these cases, contagion was measured by the successful propagation of extreme content, which may be biased by a number of factors such as user popularity, relative activity of more extreme users, and prevalence of this content. As such, whether online exposure to hate speech from other users increases the likelihood that users will adopt hate speech themselves remains unknown. In controlled environments, exposure to online content implicating outgroups as perpetrators of violence is shown to increase support for violent policies against these outgroups, as well as increasing the likelihood users will make violent comments themselves (Javed \& Miller, 2019), but whether this holds for 'real-world' online environments is unclear.

The spread of online hate is also shown to be influenced by offline 'trigger' events, such as terror attacks (Burnap et al., 2014), which can lead to the rapid spread of hate against the perceived perpetrator groups (Williams \& Burnap, 2016). This spike in online hate quickly diminishes however, and on the whole, following these trigger events, messages containing explicit hate terms are less likely to be further shared by others than messages without hateful language (Burnap \& Williams, 2015). This therefore suggests that online contagion effects may be driven by other factors, such as network position or social status.

\section{The social dynamics of hate on social media}

Given the prevalence of hate speech on social media, understanding the dynamics of how it spreads is vital for informing counter measures or mitigation strategies, however there is much still to be understood. A key question is whether hate speech expression on social media is a normative / socialisation effect or a fixed phenomenon, i.e. whether a user's propensity to express hate speech increases with time spent interacting with others within a social media platform, or whether users instead arrive with fixed views on outgroup denigration which they retain and express. This is an important question because it can provide insight into whether social media platforms are playing a driving role in the observed increases in far-right extremism by allowing hate filled conversations to take place on their platforms. Addressing this question is a key objective of this study.

There is already emerging evidence for effects of socialisation on intergroup conflict and hate speech expression on social media. For example, frequent users of Facebook have an increased perception of social distance and negative attitudes towards outgroups (Settle, 2018). On Twitter, approximately half of users who express Islamophobia change their rate of posting hate over time (Vidgen, Yasseri, \& Margetts, 2019), suggesting they may be adapting to the norms of the environment. Similarly, while hateful expressions such as Islamophobia, homophobia, or racial slurs are considered norm violations on most parts of mainstream platforms, within certain sub-communities on these platforms these expressions are considered acceptable and within 'normal' discourse, leading to much higher prevalence (Chandrasekharan et al., 2018). This high prevalence is maintained even when these groups are isolated from the wider online community (Chandrasekharan, Jhaver, Bruckman, \& Gilbert, 2020). Similarly, qualitative analysis of online forums popular with the far right shows that they are used as a space for informal social learning of political ideology and community norms, as well as more formal recruitment or operational planning (Lee \& Knott, 2020).

The nature of the language used in these more extreme environments also supports this idea, with marginal and extreme topics becoming mainstream over time (Braun et al., 2020). This shift supports ideas of contextualisation of conversations within the group, while the numerous ingroup jokes, coded language, and nebulous 'othering' present in fringe far-right platforms suggests that socialisation is 
occurring to allow for collective understanding (Tuters \& Hagen, 2018, 2019). Evidence for socialisation effects also comes through investigating the impact of censorship on hate speech expression. Suspensions of hateful forums on Reddit (subreddits) leads to an influx of users from the banned spaces on other subreddits, but does not cause significant changes in hate speech within these spaces (Chandrasekharan et al., 2017) suggesting that hate expression is driven by the community or group level characteristics of the previous group--with the caveat that many users from the banned subreddits may not migrate to other subreddits and instead take their hate expression elsewhere on the web. Similarly, users banned by Twitter for spreading hate are more popular than random users (Chatzakou et al., 2017), suggesting that they had exerted social influence on other users prior to suspension. Despite these findings, it is unclear whether social media is instantiating hateful opinions in previously non-hateful users.

Alternative evidence however suggests that users seek out online hate communities to express their pre-existing opinions, and therefore arrive into an online space with already established prejudices. This is supported by findings that users migrate from less hateful to more hateful online spaces as their own hate expression increases (Ribeiro et al., 2020), suggesting they seek out areas to express these views. Similarly, hateful users on Twitter have newer accounts than non-hateful users, supporting the idea that this hate is not driven by social interactions over time (Ribeiro, Calais, Santos, Almeida, \& Meira, 2018). Corroborating this, the relationship between exposure to extremist content on social media and self-reported political violence is strongest when individual actively seek out extremist content compared to passive encounters (Pauwels \& Schils, 2016). Overall, the role of socialisation in driving hate expressions remains therefore unclear.

These ideas need not be mutually exclusive however, and it is possible that users could arrive into a social media platform with existing prejudices and also develop these further through interactions with other users. This duality is demonstrated in studies of Reddit. Hateful subreddits have been shown to be fairly stable in their levels of hate over time, suggesting that the norms of the groups are fixed (Grover \& Mark, 2019). Additionally, users on Reddit are shown to engage in a process of preentry learning prior to posting in a new subreddit, and to moderate the toxicity of their language to match that of the community that they are joining (Rajadesingan, Resnick, \& Budak, 2020). This suggests that socialisation effects may occur prior to posting messages. However, the shift in language observed in those users does not affect their posts within other communities on the platform. This suggests that these users may also be seeking out these hateful communities to express hate which they would not share in more moderate spaces. More research is therefore needed to understand the role of hateful social media environments in driving user individual expressions of hate speech, whilst accounting for users' initial positions upon joining the platforms.

\section{Secondary transfer effect of hate}

If exposure to hate speech increases the likelihood that individuals will express hate themselves, an important question arising is whether this newly expressed hate will remain specific to the targeted group in the original hateful message(s) which the new expresser was exposed to, or whether this hate can spread to wider targets. Offline, increased contact between opposing groups is shown to reduce prejudice and conflict (Allport, 1954; Brown \& Hewstone, 2005; Pettigrew \& Tropp, 2008). This effect is not limited to the groups involved directly in the contact, but rather that there is a secondary transfer effect by which contact with a primary outgroup reduces prejudice toward other (secondary) outgroups (Pettigrew, 2009; Schmid, Hewstone, \& Tausch, 2014; Tausch et al., 2010).

This secondary transfer effect occurs in part due to attitude generalisation - a process whereby attitudes towards one group generalise to another linked but less familiar group (Fazio, Eiser, \& 
Shook, 2004) - and also due to ingroup reappraisal, whereby individuals realise that the specific ingroup norms they are familiar with are not the only acceptable set of social rules (Pettigrew, 1997). Together these effects give rise to the transfer of positive contact effects across groups (Tausch et al., 2010). For example, contact with foreigners has been shown to lead to more positive attitudes towards this outgroup, but this positive effect also generalises towards other much wider outgroups that were not involved in the original contact situation, such as the homeless or LGBTQ+ groups (Pettigrew, 2009).

While much of the research in this field has focused on the constructive outcomes of positive intergroup contact, there is increasing evidence for a similar effect in reverse, with negative intergroup contact leading to more generalized negative outgroup attitudes (Jasinskaja-Lahti et al., 2020; Meleady \& Forder, 2019). In the context of online hate speech, it is therefore possible that increased exposure to the negative attitudes of ingroup members towards one outgroup will lead to worsening attitudes against both this target outgroup and more widely against other outgroups. This may occur through an effect of generalised outgroup prejudice, or through a reduction/reversal of the ingroup reappraisal process, whereby the social norms of the ingroup are viewed as the only 'correct' or acceptable situation. To our knowledge this has yet to be tested.

\section{The current research}

This study aims to test whether participation in online conversations containing hate speech increases the likelihood that users will adopt the hateful norms of the group, through a process of social contagion, and whether users express greater levels of hate speech themselves as they spend longer on the platform.

We address this question using a fringe social media platform popular with the far-right and known to have high levels of hate speech, Gab. We construct our analysis in three steps. First, we identify hate speech in online conversations using a supervised machine learning approach, and determine the targeted group of this hate. Second, we build a picture of the overall trajectory of hate speech expressions for users on the platform by calculating the likelihood of a user expressing hate speech as they post successive messages. Finally, we use network contagion models to look at the structure of the network (i.e. which users are interacting with one another) and measure the network-wide patterns of user exposure to, and expression of, hate speech.

\section{Gab - An ideal platform to study hate speech contagion}

In this study we analyse messages from Gab, is a micro-blogging platform designed as a broad copy of Twitter (Benson, 2016; Weissman, 2016). Users post short messages which can be reposted, replied to, or 'liked' by other users of the platform. Gab is known for its lax hate speech moderation policies and the high proportion of messages containing explicit hate terms (Lima et al., 2018; Zannettou et al., 2018), and is seen as 'rolling out the welcome mat' to users banned from social media sites (AntiDefamation League, 2019). The platform is heavily focused on political content and topics closely follow current affairs, particularly around political ideology, race, and terrorism (Zhou, Dredze, Broniatowski, \& Adler, 2019), including Nazi imagery, antisemitic material, holocaust denial, islamophobia, along with much wider forms of hate against other target groups (Weich, 2019). In addition, the platform has been used as a recruitment tool by several neo-Nazi and alt-right groups (Katz, 2018), and the rise in popularity of extremist far-right groups on Gab has been suggested to be "remarkably similar" to the rise of ISIS on mainstream social media in prior years (Makuch, 2019). In October 2018 Gab was taken offline after the shooter at the Tree of Life Synagogue attack, 
Pittsburgh, USA, was found to have posted anti-Semitic messages on Gab immediately prior to the attack and received substantial support from platform members (Mathew et al., 2019).

As the platform has hosted conversations which became increasingly problematic up to a point of extreme offline violence, it is a valuable case study in online hate. The high prevalence of hate speech additionally means that exposure to this type of content will be much higher than on mainstream platforms, with larger samples of hate messages allowing for more reliable estimates and more statistical power. In addition, Gab has been shown to have low levels of algorithmic filtering and artificial promotion of content compared to other social media platforms (Reed, Whittaker, Votta, \& Looney, 2019). This makes Gab a valuable platform to study the effects of social exposure to content on online behaviours because users' connections on the platform are responsible for the patterns of content they are exposed to, rather than artificial promotion of content or algorithmic filtering (O'Callaghan, Greene, Conway, Carthy, \& Cunningham, 2015). Finally, the networked structure of the platform, akin to Twitter networks, means that content can theoretically propagate across the entire network and is not restricted to specific pages or groups as would be the case on a more compartmentalised platform such as Facebook, allowing us to investigate network effects more easily.

\section{Research aims}

This study aims to provide new insight into how hate develops on social media platforms.

More specifically, our first objective is to investigate whether users seek out social media platforms to express pre-established hate, or whether hate speech expression arises as users spend more time within these platforms. Based on theories of group extremism we hypothesise that users will increase their hate speech expressions over time spent on the platform.

Our second objective is to test whether online hate speech spreads through social contagion. Based on prior work on online informational and emotional influence we hypothesise that social contagion will occur and therefore expect to find that users will be more likely to express hate speech after having been exposed to it from other users.

Finally, we will aim to explore these effects of hate speech contagion in more detail, by testing (i) whether its effects are specific to certain hate types or whether they transfer prejudice across hate types, and (ii) at what temporal scale this effect occurs. 


\section{Methods}

This study uses a combination of natural language processing, multi-level general additive modelling, and network analysis to investigate how the hate speech expressions of users on fringe social media platforms vary with time spent on the platform.

\section{Data collection and pre-processing}

We analyse all the messages from Gab posted between its formation on $10^{\text {th }}$ August 2016 and the $29^{\text {th }}$ October 2018 when the platform was taken offline following a terror attack committed by one of its users. This dataset contains 33,089,208 messages posted from 259,598 different accounts. This period consists of an amalgamation of data shared by (Zannettou et al., 2018) which covers August 2016 January 2018 and data from the online repository Pushshift which covers the remainder of this period from January 2018 - October $29^{\text {th }} 2018$.

To prevent spam or automated 'bot' accounts from influencing of the results, we identified and removed accounts which made $>10,000$ posts over the entire period (450 accounts). This coarse method may not have removed all automated accounts, but fully detecting automated activity was beyond the scope of this study. Importantly, we included these accounts when making platform-wide calculations on the level of hate present on the platform each day, as this can be affected by inauthentic activity (Gallacher \& Heerdink, 2019), but excluded them from all other user-level calculations.

\section{Automatic detection of hate speech in social media posts}

Hate speech is defined as messages which express hatred towards a targeted group with the intention to be derogatory, to humiliate, or to insult members of that group. Specifically, a message requires three features to constitute hate speech: the message must be (1) a deliberate attack, (2) directed towards, or about, a specific group of people, and (3) motivated by, or focused on, aspects of the group's identity. ((Davidson et al., 2017; de Gibert et al., 2018; Gallacher, 2021). This definition is broad enough to contain any targeted group and is not restricted only to specifically protected groups and characteristics (See Gallacher, 2021, for more details).

We identified hate speech in the dataset using a supervised machine learning approach with extrapolates human annotations on a random sample of messages to the entire dataset. The model we used leverages cross-platform datasets of hate speech along with pre-trained contextualised word embedding models (BERT) to encode semantic information along a number of manually engineered syntactic features commonly associated with hate expressions (see details in Gallacher, 2021). These features include the message length, the presence of explicit hate terms, hate symbols, and obscenity, the lexical density, the presence of 'othering' and the sentiment (positive vs negative) of the message. This model is trained on a total of 40,000 messages from Facebook, Twitter, Stormfront, and Gab. The data from these four individual platforms is used to train four platform-specific models, which are then combined into one model using a 'superlearner' approach (the superlearner 1.0 outlined in Gallacher, 2021). These platforms were chosen as they represent a broad range of presentations of hate speech, and range from the mainstream to more fringe areas. By combining data from multiple platforms, this model outperforms a model trained on any one single platform in isolation. This approach retains platform specific hate characteristics however, and therefore aspects of hate expression which are specific to Gab should be retained and detected by the model.

We used this approach to classify all messages in our datasets into 'clean', 'offensive' and 'hate speech' (as defined above) categories and obtained a confidence score for each message. Offensive 
messages are broadly defined as messages likely or with the intention to cause offence (Davidson et al., 2017). This includes uncivil, rude, inappropriate, or overly disrespectful content. Often this content contains obscenity and profanity, although the presence of these words does not necessarily signal malicious intent or group directed abuse (see Gallacher 2021 for further details). To minimise the impact of false positives in the subsequent analysis, we re-classified any message with a low confidence score as 'clean', as this is the majority class in the dataset. This approach performed well for Gab data, with an overall accuracy of $90.9 \%$ when measured on a representative sample of the Gab messages used in this study. The precision and recall scores for hate speech in particular were 0.75 and 0.83 (see Gallacher, 2021 Supplementary Information (SI) Table 3 for full performance figures and Gallacher, 2021 Figure 5 for the confusion matrix). The lower values on these measures when compared to overall accuracy reflect the fact that hate speech is a minority class on these social media platforms, and the majority of messages do not contain hate. Given this, a precision score of 0.75 (one of four messages flagged as hate speech will be done so incorrectly), represents a reasonable level of performance, and favourable in relation to comparable models (e.g. Davidson et al., 2017; Kennedy et al., 2020).

In addition to the classification for each message, we also extracted and stored a number of semantic and syntactic linguistic features which were used for this classification, so as to compare how the

Table 1: The additional semantic and syntactic features extracted for each message to investigate how the nature and presentation of hate messages changed over time.

Feature

Description and Implementation

Othering

Detection of the use of two-sided pronouns that contain a distinction between the ingroup and out-group in a single message (e.g. your/our, them/us, they/we), calculated using dictionaries of popular personal pronouns for both the in-group and out-group. Binary measure $(0 / 1)$

Hate Severity The average severity score for the hate terms in the message (range 0-100). Calculated using a dictionary approach from a database maintained the Anti-Defamation League $(\mathrm{ADL})$

Hate Terms

Hate Symbols The number of hate terms present, calculated using a dictionary approach from the ADL

The number of hate symbols present in the message, calculated using the ADL hate database

Obscenity

The number of swear words, calculated using a dictionary approach

Sentiment

The sentiment (positive / negative polarity) of the message, calculated using the qdap implementation in R. (range -1 to +1 )

Lexical Density

Measure of syntactic complexity, calculated as the ratio of content words to total words (range 0-100)

Flesch-Kincaid

Reading Ease

A measure of message readability in relation to the projected education level required to understand the message (range 0-100)

Post Length

Count of the number of words in the message

Punctuation

Count of the punctuation symbols in the message

Negation

The number of negation words present in a message, calculated with qdap implementation in $\mathrm{R}$

Pronouns approach 
nature of hate speech changes over time. These linguistic features are given in Table 1. Further details of how these features are calculated and their relation to hate speech are given in Gallacher, 2021.

\section{Target of hate detection}

To identify the type of hate speech expressed in the messages, i.e., the group targeted, we used guided topic modelling (Anoop, Asharaf, \& Deepak, 2016; Li, Chen, Xing, Sun, \& Ma, 2019). This combination of supervised machine learning to detect abuse and unsupervised machine learning to identify the nature of the abuse has proven successful before (Agarwal \& Sureka, 2015; Theocharis, Barberá, Fazekas, \& Popa, 2019), and can lead to superior performance over training specific supervised classifiers for each individual hate type (Park \& Fung, 2017).

\section{Topic modelling}

To perform this topic modelling we used a form of semi-supervised machine learning called Guided Latent Dirichlet Allocation (LDA) (Blei, Ng, \& Jordan, 2003). This approach treats each message as a mixture of topics, and each topic as a mixture of words from within all of the messages in the dataset. This allows messages to "overlap" each other in terms of content, rather than being separated into discrete groups, in a way that mirrors typical use of natural language (Silge \& Robinson, 2017). Different types of hate speech (e.g., anti-Semitism, Islamophobia, homophobia) will form different topics within the hate speech messages and we can use this distinction to identify one type of hate from another. We trained the LDA models on the dataset of hate speech messages from the same four social media platforms (Gab, Facebook, Twitter, and Stormfront) used to train the earlier hate speech detection model to ensure consistency. The steps in this process are shown in SI Figure 1.

Prior to performing the LDA we pre-processed the text to remove stop words, extract unigrams and bigrams, and then used an information retrieval approach to increase the co-occurrence of words within the messages and improve consistency and performance by identifying the most similar messages in the dataset and combining them for model training (see details in SI 1.2). The optimum number of topics for the LDA model was calculated dynamically using a range of model performance criteria (Nikita, 2016) (see SI 1.2 \& SI Figure 2). This process identified 11 distinct hate speech topics.

To improve the consistency and distinctiveness of these topics we introduced a subsequent guided step (Anoop et al., 2016; Li et al., 2019), whereby we manually identified the most salient words per topic, and input these as seed words to retrain the model to help anchor the topics towards these areas. These words were then weighted higher in topic creation, to improve both topic-word distributions (by biasing topics to coalesce around appropriate seed words) and to improve document-topic distributions (by guiding documents towards topics related to the seed words they contain). This has been shown to improve the performance of unsupervised topic modelling by adding subject matter knowledge into the process and by guiding the topic models to converge in meaningful directions (Jagarlamudi, Iii, \& Udupa, 2012). For example, when racial slurs are used in place of actual group names, the slur and the group name will not co-occur naturally, but by guiding them manually to the same topic they can be placed together. Equally, words which often co-occur but form different topics may be placed into the same category incorrectly in classic topic modelling. Adding these two words as seeds into different topics can help prevent this from occurring. At this stage we re-ran the LDA for the same number of topics, this time guided towards the identified topics (SI Figure 1 stage 2.3). The final topics that the model produced are shown in in table 2 . 


\section{Topic model performance}

We measured performance of this hate topic classifier by taking a random sample of 100 messages from each topic and manually identifying whether these topic judgements were correct. These accuracy measurements for each topic are shown in table 2 . These judgements were independently checked by a second coder. Intercoder reliability scores gave a percentage agreement of $89.4 \%$, a Krippendorff's Alpha of 0.717, and a Cohen's Kappa score of 0.720. The former is at the level of 'good' agreement (George \& Mallery, 2003) while the latter is well above the 0.61 threshold for reliable coding and substantial agreement (Glen, 2014), and so we retained our labelling as accurate.

The accuracy in detecting the type of hate speech varied greatly between topics. Anti-Semitism, antiBlack racism, and anti-immigration sentiment were the most accurate topics, with accuracy scores ranging from $90.0 \%$ to $81.0 \%$ (Table 2). These are high accuracy scores compared to the noinformation rate of this classifier of $9.1 \%$ (assuming random distribution of these 11 topics). Accuracy in detecting other forms of hate speech, such as hate directed towards Mexicans and Liberals, was lower, although still higher than chance. Based on these results we retained the most accurate 6 classes of topic models: anti-Semitism, anti-Black racism, anti-immigration, Islamophobia, homophobia and misogyny. All other classes were re-classified into the 'other/unknown' category. Figure 1 shows the top 20 terms for each of these six retained topics. These six hate types have been previously highlighted as the most prominent online by a UN report on online hate (Ischinger, 2020), and as the primary groups targeted by the online far-right (Conway, 2018).

DISCLAIMER - Hateful terms included in the figure below
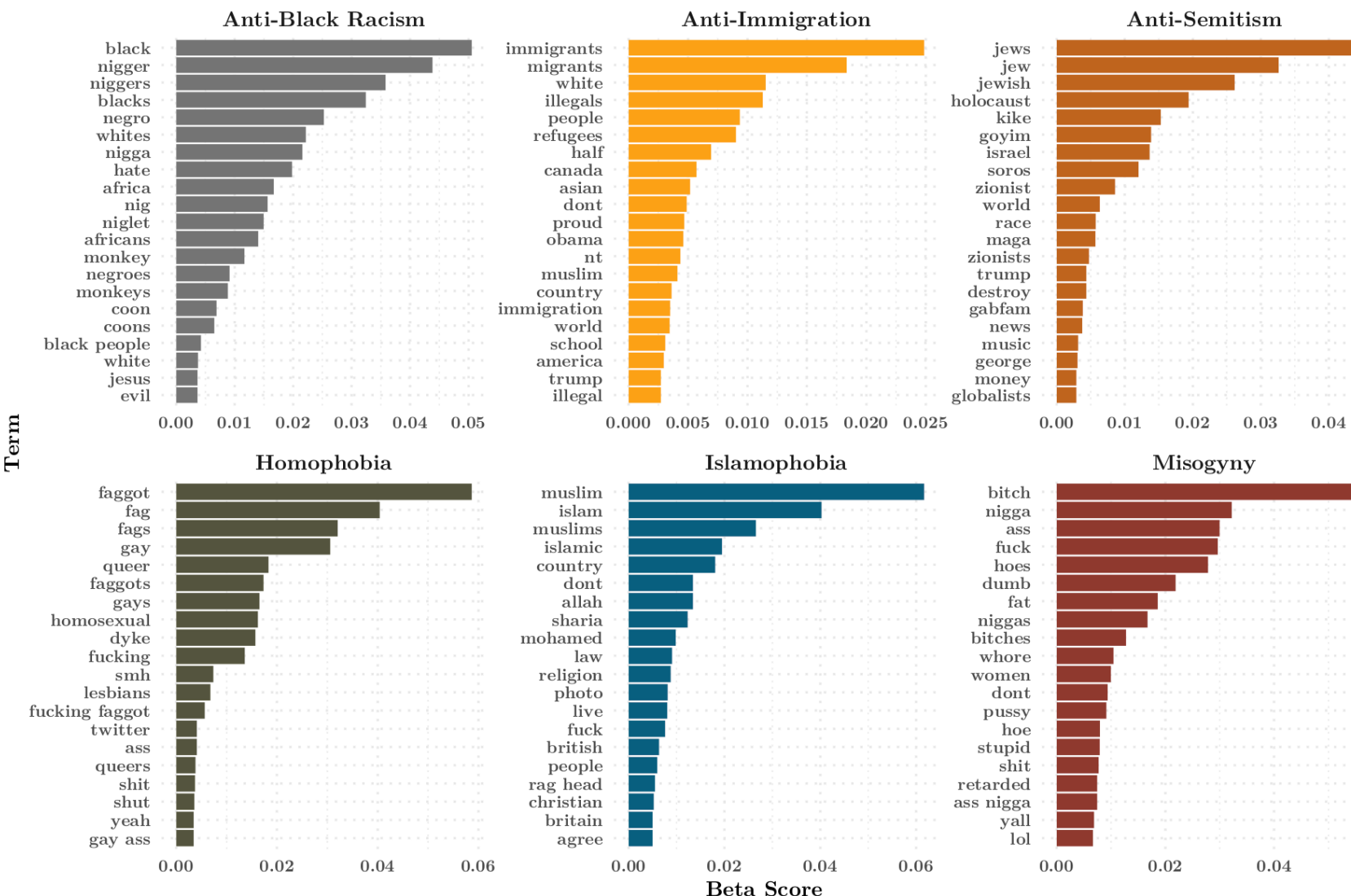

Figure 1 - The top 20 terms across the 6 retained hate topics from the guided LDA model 
Table 2 - Hate topics and accuracy of classification

\begin{tabular}{|c|c|c|}
\hline Topic & $\begin{array}{l}\text { Accuracy } \\
(\%)\end{array}$ & \multirow[b]{2}{*}{4} \\
\hline Anti-Semitism & 90.0 & \\
\hline Anti-Black racism & 89.6 & \multirow{6}{*}{ Retained } \\
\hline Anti-Immigration & 81.0 & \\
\hline Islamophobia & 73.7 & \\
\hline Homophobia & 72.2 & \\
\hline Misogyny & 71.8 & \\
\hline Anti-left hate & $--\overline{62.3}$ & \\
\hline White Supremacy & 53.0 & Re-coded to 'other' \\
\hline Anti-Mexican racism & 31.0 & \\
\hline White Trash hate & 23.0 & \\
\hline Unknown / Residual Error & NA & $\nabla$ \\
\hline
\end{tabular}

\section{Analysing the dynamics of hate speech expression}

To investigate the relationship between time spent on the platform by a user and their hate expressions (Objective 1), we calculated the correlation between users' total number of posts and the proportion of their messages which contained hate speech. To account for periods of inactivity or 'lurking', we also performed this analysis for the overall time they spent active on the platform (number of days between their first and last post). We used Spearman rank correlation as variables were not normally distributed.

\section{Measuring hate trajectories}

We further investigated the nature and trajectory of user's hate speech expressions (Objective 1) over time with multi-level general additive models (GAMMs) implemented in the 'mgcv' package (Wood, 2019). GAMMs are a class of statistical models where the relationship between the independent and dependent variables is represented by non-linear smoothing functions to capture non-linearities in relationships between variables. We modelled how the probability that a user expressed a hate message changed over their posting period within the platform (their 'hate trajectory'). We accounted for inter-user differences by including user ID as a random effect. As the overall proportion of hate messages varied greatly between days (SI Figure 3), we controlled for the overall level of hate expression on each day (the proportion of all messages on that specific day which were classified as hate speech) to observe how individual behaviour changed over time rather than the general level of hate speech on the platform.

Only $5.9 \%$ of the users posted beyond 250 posts, so to conserve good sample sizes we truncated users posts at the $250^{\text {th }}$ post. Users who posted hate to the platform but made fewer than 250 contributions overall had their full post history included. Users who never posted hate on the platform were excluded from this analysis as it would not be possible to assign them a hate trajectory. Following this filtering we retained $24.7 \%$ of posts from the dataset $(8,173,034$ posts). For robustness, we also ran the same analysis over a longer post history, including either the first 2150 posts from each user or their entre post history, whichever was longest. This 2150 threshold was chosen as $99 \%$ of users did not post past this point). The posts included represented $59.7 \%$ of overall posts. 


\section{Changes in the nature of hate expression}

In order to test whether the style of hate expressed by users changed over time as they spent time on the platform, we firstly compared the linguistic properties (metrics detailed in table 1) of their first hate post to the average of the inter quartile range (IQR) of their subsequent hate posts. Comparing the first message to the average of the IQR controls for the different number of hate speech posts each user makes, and for each user we are therefore comparing their first message against their latter messages, regardless of how long or how many messages they contributed to the platform overall. For example, for a user who posted 15 messages in total, we compared their first message to the average of their $4^{\text {th }}$ to $12^{\text {th }}$ messages. Conversely, for a user who posted 100 messages, this would be comparing their first to the average of their $25^{\text {th }}$ to $75^{\text {th }}$ messages.

To get a further measurement of how users' hate expressions changed over time, we also counted the number of different groups targeted by hate speech that users expressed in their early (first 100) and compared this to their subsequent $\left(101^{\text {st }}-200^{\text {th }}\right)$ messages.

\section{The effects of hate message engagement}

To measure the role social feedback plays in these hate speech trajectories, we tested how the number of reactions (engagement) received by a hate speech post affected the likelihood of the user who posted the message posting subsequent hate messages. The number of reactions was measured as the sum of the 'Likes' and 'Dislikes'. We did not differentiate between the nature of these responses as it is difficult to distinguish between endorsements and opposition in these options, and likely it depends on the nature of the message. For completeness, we also compared the average number of responses that hate speech messages received compared to offensive and clean messages. These values were normalised due to highly right-skewed distributions (i.e. many messages receiving few reactions, and a few receiving a very high level of engagement).

\section{Measuring network contagion and exposure to hate speech}

In order to measure network contagion effects of hate speech spread (Objective 2), we first preprocessed the data to create daily conversation networks containing dynamic information about the spread of hateful messages between users on that day. For each day in the dataset we identified all users taking part in the conversation, and the interactions between them. These interactions can be replies to others' messages, new messages directed at other users through mentions (@mentions), or reposts of other users' prior messages. We did not differentiate between these types of interaction. We recorded the time each interaction took place. For each user, we then identified the time (hour) within that day when they first posted hate speech, which represents their time of 'adoption' of hateful behaviour for that day. We found that the Gab conversation followed a strong daily rhythm with a peak of activity between $1600 \mathrm{hrs}$ and $2000 \mathrm{hrs}$ (Eastern time zone, UTC-5) and a minimum at 0700hrs. This supports the separation of the conversation into daily networks, however to minimise conversation split across days, we therefore used $0700 \mathrm{hrs}$ as the boundary between two days to separate daily conversations, rather than $0000 \mathrm{hrs}$ which is near the peak activity (see details in SI Figure 4).

With this information, we constructed the dynamic conversation network between users, with weights included for multiple connections but without self-loops (Figure 2a). To filter out peripheral activity and isolated nodes, and ensure consistency and computational efficiency, we performed a k-core 
A)

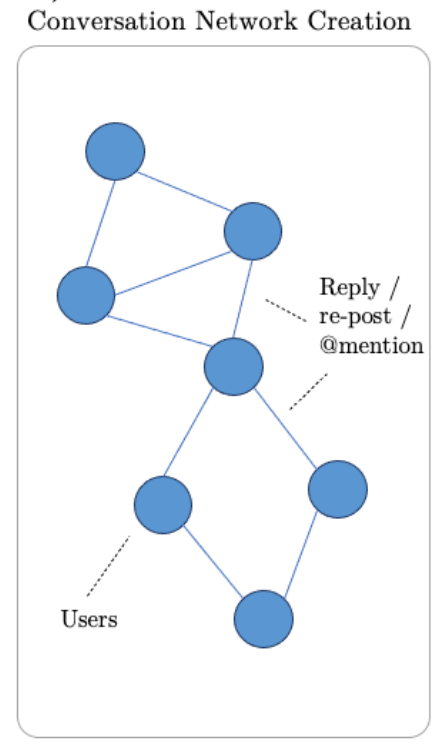

B) Hate Speech Exposure Estimation

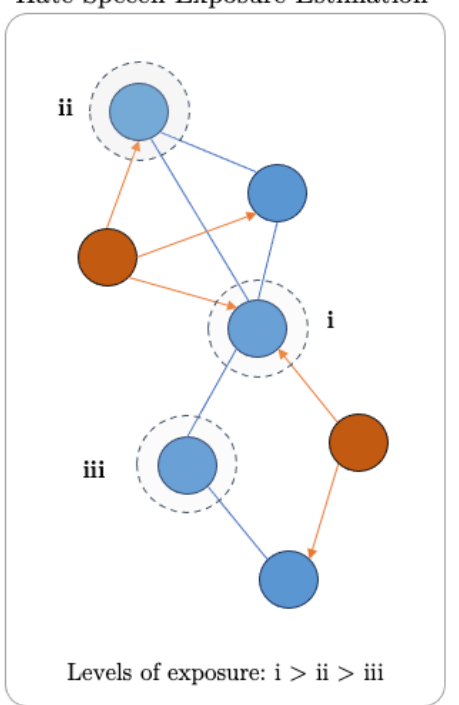

C)

Measurement of Social Contagion Effects

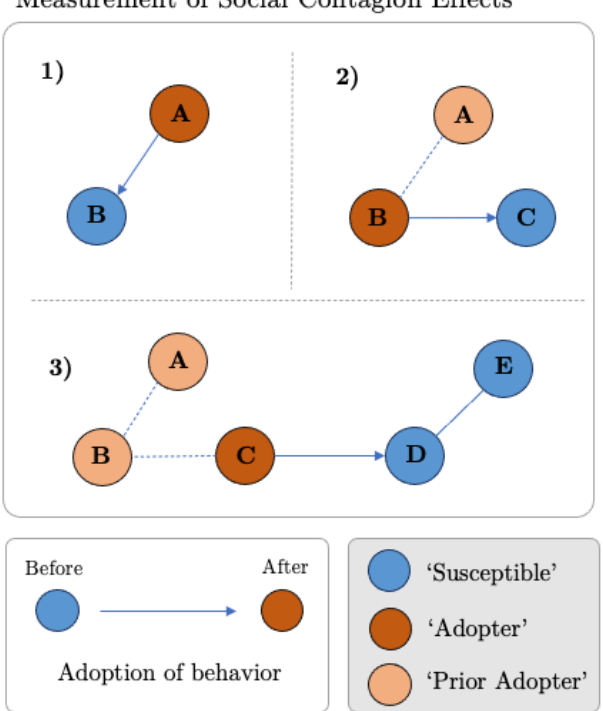

Figure 2 - Creation of the conversation networks and measurement of exposure and contagion effects. Here, adopting the behaviour represents expressing hate speech (dark red), 'susceptible' individuals have not yet expressed hate speech (blue), and 'prior adopter' individuals have but cannot contaminate nor become contaminated again (orange).

reduction with a threshold of $\mathrm{k}=2$. This means that all remaining users have connections to at least two other users on that day.

This network was then made dynamic using the NetdiffuseR package (Valente, Dyal, Chu, Wipfli, \& Fujimoto, 2015; Valente \& Vega Yon, 2020; Vega Yon, Valente, Dyal, \& Hayes, 2020), with each day treated as a different conversation network and with each hour a 'slice' of that daily conversation network. This allowed us to calculate the 'exposure' to hate speech that each user received at each hour. Higher exposure occurs if the users are closer to users in the network when these users express hate speech (Figure 2b). Specifically, the exposure to hate speech was calculated as the proportion of each users' connections who adopted the behaviour (i.e. expressed hate speech) in that time period, taking the weights of the connections into consideration (Valente, 1996). This value is then normalised between 0 and 1, with higher scores reflecting higher levels of exposure. Network exposure is measured on direct contact (rather than structural equivalence) and therefore captures social influence conveyed through overt transmission of information, persuasion or direct pressure (Valente, 1996). Social contagion effects will be observed in these networks if users are more likely to express hate speech after having previously been exposed to it by other users (Figure 2c).

\section{Testing for the effect of network structure - short-term vs longer-term hate contagion}

We tested the social contagion effects for hate speech (Figure 2c) at two levels: in the short-term and in the longer term (Objective 3).

At the short-term (hourly) level we looked at hourly network slices (over all days) and tested whether network exposure to hate speech in hour $h$ was associated with a greater likelihood of subsequent hate speech expression during the next hour $h+1$. For this we used a logistic regression model (generalised linear mixed model, GLMM) with user ID and date as random effects to control for differences between users and changes within the platform as a whole over time. We also added hour of the day 
(1-24) as a fixed effect to control for any effects of certain times of day containing greater hate speech expressions. A caveat of using one-hour slices is that if users are exposed to hate and then post it themselves in the same hour, we will miss this behaviour, however looking at sub 1-hour slices is not feasible due to the computational demands caused by long-term nature of the dataset. On the other hand, our method means that we are unlikely to detect users simply echoing others' sentiments in the direct replies, as these would likely occur within the same one-hour network slice.

At the longer-term level we tested for relationships between network exposure to hate speech and expressions of hate speech that existed across multiple days. For each user, on each day, we calculated their exposure to hate speech on that day up to the point of posting hate speech themselves (if they didn't post any hate, then their exposure would be calculated over the whole $24 \mathrm{~h}$ period).

Additionally, we calculated the average daily exposure to hate speech that they received over the preceding 7 days. For each day, the response variable was a binary indicator of whether they posted hate that day or not. This longer-term approach also allows us to account for another caveat of the short-term approach. Due to the nature of the diffusion network models within NetdiffuseR, it is not possible to include multiple times of adoption for a behaviour by the same user within the same network, and so we are only measuring exposure effects for the first time a user expresses hate speech on each day. It is therefore possible that we are missing cumulative effects of users expressing hate speech repeatedly within a day, leading to greater exposure of their contacts. Looking at longer-term effects over multiple days allows us to address this issue.

For these long-term models we again used GLMMs and tested simultaneously for the effects of both daily and weekly exposures with user ID and date as random effects, but additionally including the cumulative number of days that users were active up until the day of posting as random effect, to account for any effects of platform use on increasing likelihood of posting hate speech. Model performance for short and longer-term effects was compared using Akaike Information Criterion (AIC). By controlling for users' exposure to hate speech at the short-term level within these longerterm models, we are separating any immediate effects of conversation topic clustering from longerterm exposure. This is important, as clustering (aka homophily; the tendency for similar users to group together e.g. Barberá, 2015; McPherson, Smith-Lovin, \& Cook, 2001) and contagion are easily confounded in observational studies of contagion effects (Shalizi \& Thomas, 2011). These clustering effects can be assumed to be equal across days, so can be controlled for by controlling for daily conversation exposure.

\section{Secondary transfer effect}

To test for transfer effects of hate speech exposure across hate types, we performed a similar analysis at both the short and longer-term level separately for each specific hate type identified by the topic modelling. We calculated each user's exposure to specific types of hate at each network slice, along with their adoptions of these specific hate types. We tested for the predictive power of exposure to each hate type on the adoption of each hate type with similar GLMMs as above, in each model including exposure to all other hate types to control for these effects. To control for the effects of multiple comparisons, Holm's corrections were applied to the obtained p-values.

\section{Ethics}

All research was conducted in accordance with the University of Oxford Ethics Committee (Ethics Reference: SSH_OII_CIA_19_062). All data collection was conducted using open source methods and publicly available data, and hence, informed consent was not explicitly obtained. No private groups were joined, and no accounts 'befriended' in order to access data that are not publicly 
available. In order to preserve anonymity, we took a cryptographic hash of all usernames prior to analysis and real account usernames have not been used in the analysis at any point.

\section{Data analysis}

All analysis was done in R (version 3.6.1) using the tidyverse (Wickham et al., 2019) and tidymodels (Kuhn, Wickham, \& RStudio, 2020) collections of packages. Where particular additional packages have been used, they have been referenced in the text. Where applicable, summary statistics are presented as mean $+/$ - standard error.

\section{Results}

Overall, $8.9 \%$ of messages on Gab during the period August 2016 - October 2018 were classified as hate speech, with $28.9 \%$ of users expressing hate speech at least once when active on the platform. For users who expressed hate, Islamophobia was the most common type of hate speech (39.4\%), followed by anti-immigration sentiment (36.0\%) and anti-Semitism (15.5\%) (Table 3, Figure 3). Many users shared multiple types of hate, with $18.2 \%$ of hate expressing users sharing a combination of Islamophobia and anti-immigration sentiment, while $7.9 \%$ of these users shared a combination of Islamophobia, anti-Semitism, and anti-immigration sentiment and $10.3 \%$ shared all six types of hate.

Together these six hate types formed $61.4 \%$ of the overall hate speech within Gab over the study period, with the other hate types (plus unidentifiable hate messages) making up the remaining $38.6 \%$ of hate speech messages.

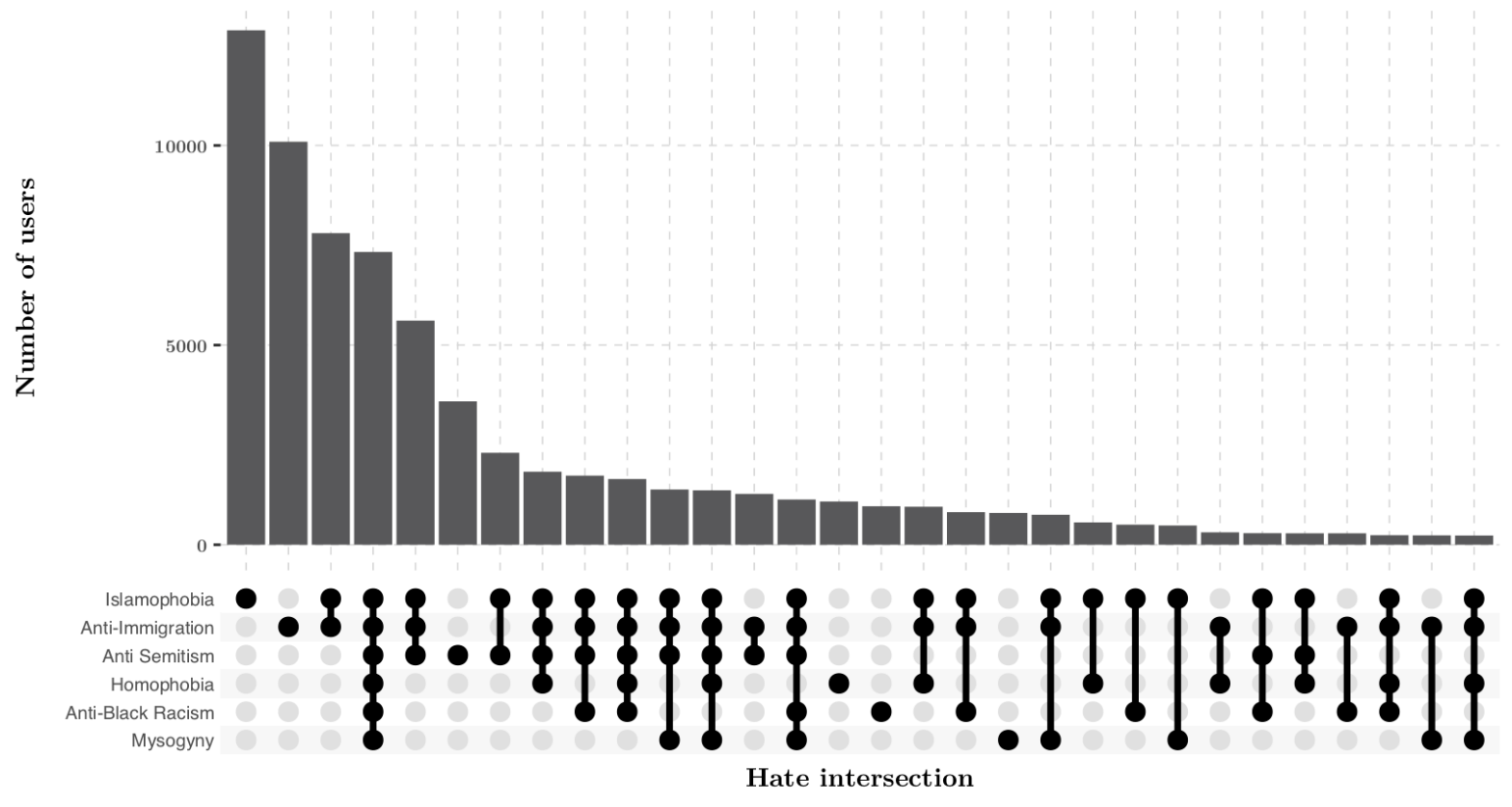

Each column represents unique combinations of hate types

Figure 3 - The frequency of common intersections of types of hate speech expression across hate types. Columns show the number of users who uniquely shared this combination of hate types, with the intersections given below. 


\section{Users' hate speech expression over time}

We found a positive relationship between users' level of activity on Gab and the proportion of hate speech in their posts. Both users' total number of posts and the duration of their active period on the platform were positively correlated with the proportion of their posts expressing hate (Spearman's Rho, number of posts: $r_{s}=0.562, \mathrm{p}<0.001$, active period: $\left.r_{s}=0.864, \mathrm{p}<0.001\right)$

When investigating users' trajectories of hate speech expression, we found a high likelihood of users posting hate early, with $10.8 \%$ of users expressing hate in their first post upon joining the platform. This is higher than expected by chance if all hate posts were evenly distributed (8.9\%). The number of subsequent posts had a large impact on the likelihood of hate expression, and followed a non-linear relationship over the subsequent 250 posts (GAMM, $\mathrm{n}=8,173,034, \mathrm{df}=7.259, \mathrm{~F}=5.061, \mathrm{p}<0.001)$. Initially, the likelihood of expressing hate speech decreased as they started to take part in the conversations, but this trend later reversed, and the likelihood of hate expression increased with every subsequent posted message. The turning point of this trajectory occurred around the $25^{\text {th }}$ post (Figure 4). Over a longer period of up to 2150 posts on the likelihood of a user posting hate speech increased until around the $1000^{\text {th }}$ post before levelling off (SI Figure 5a, GAMM, $\mathrm{n}=18,159,111$, $\mathrm{df}=1.533, \mathrm{~F}$ $=153.3, \mathrm{p}<0.001)$.

To control for the possible artefact of more moderate users leaving the platform early, and driving the latter stages of these user hate trajectories, we additionally (i) calculated 'hate trajectories' over the log of user post numbers to minimise the impact of fewer users being active over longer periods and (ii) looked at the hate trajectories for the most active users (with $\geq 250$ posts) only. The results from these robustness checks showed that trajectory was not caused by moderate users leaving the conversation; looking at the hate trajectories over the log of user post numbers gave a similar ushaped trajectory (SI Figure 5b). The hate trajectories for just those users who posted $>250$ messages followed an upwards trajectory over time (SI Figure 6a\&b).

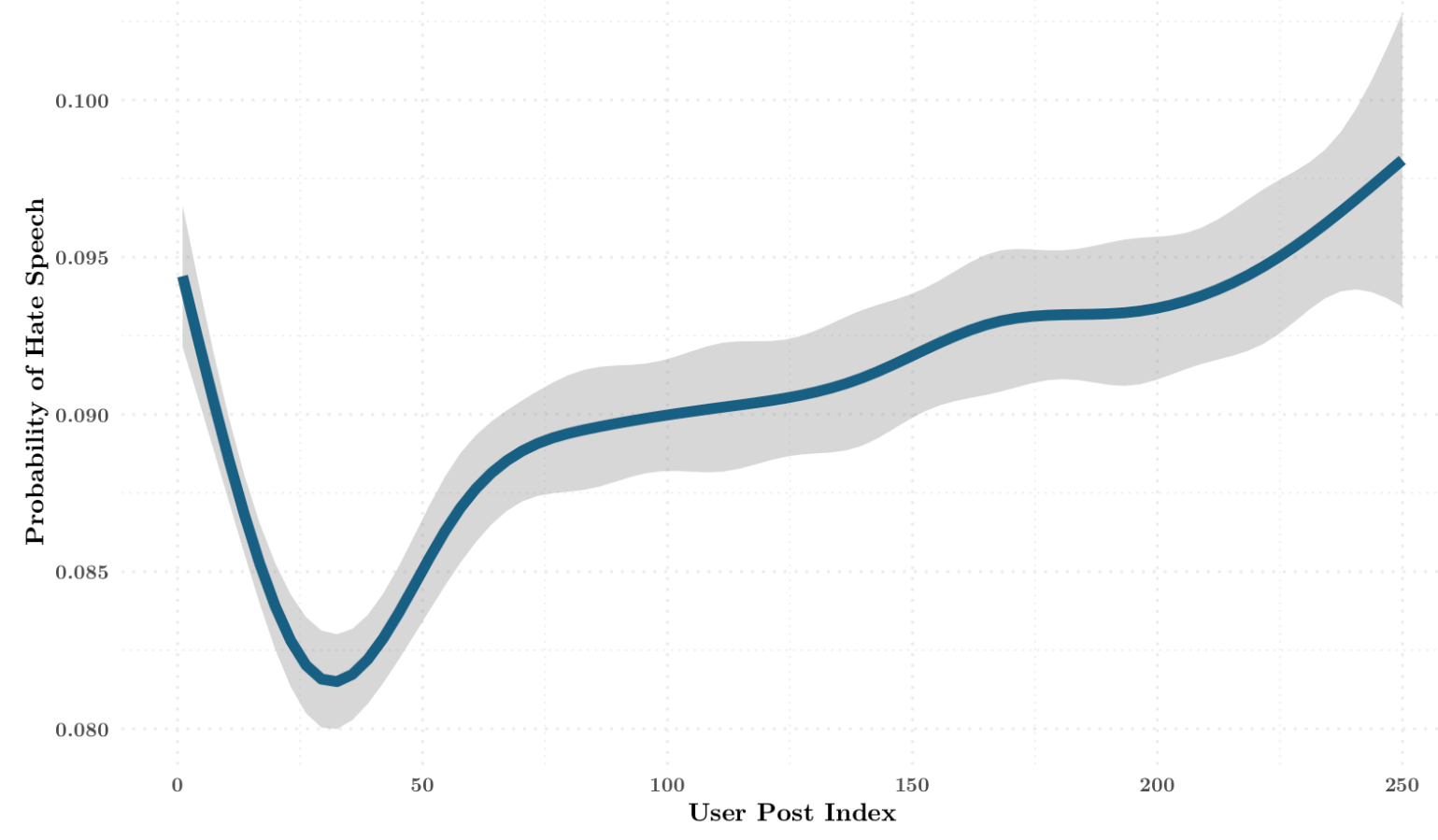

Figure 4-Overall user hate trajectories for Gab for the first 250 posts users made to the platforms 
Table 2 - Differences between initial hate speech messages and later (average of Interquartile Range, IQR) hate speech messages for users within Gab (means +/-se)

\begin{tabular}{|c|c|c|c|c|c|}
\hline Metric & $\begin{array}{c}\text { First hate } \\
\text { post }\end{array}$ & se $(+/-)$ & $\begin{array}{c}\text { Later hate } \\
\text { posts } \\
\text { (IQR) }\end{array}$ & $\begin{array}{c}\text { se } \\
(+/-)\end{array}$ & Difference \\
\hline $\begin{array}{l}\text { Daily Hate Density } \\
(0-1)\end{array}$ & 0.084 & 0.000 & 0.091 & 0.001 & -0.007 \\
\hline Post Length (words) & 34.530 & 0.064 & 34.57 & 0.401 & -0.042 \\
\hline $\begin{array}{l}\text { Hate Severity } \\
(0-100)\end{array}$ & 9.530 & 0.110 & 8.041 & 0.585 & +1.489 \\
\hline No of Hate Symbols & 0.023 & 0.001 & 0.028 & 0.005 & +0.005 \\
\hline No of Hate Terms & 0.171 & 0.002 & 0.154 & 0.012 & +0.017 \\
\hline $\begin{array}{l}\text { Lexical Density } \\
(0-100)\end{array}$ & 40.160 & 0.056 & 40.36 & 0.416 & -0.203 \\
\hline No of Negation terms & 1.112 & 0.005 & 1.109 & 0.035 & +0.003 \\
\hline No of Reactions & 33.163 & 0.364 & 32.55 & 2.305 & +0.614 \\
\hline No of Obscenity terms & 0.467 & 0.004 & 0.390 & 0.022 & +0.076 \\
\hline $\begin{array}{l}\text { Othering } \\
(0-1)\end{array}$ & 0.134 & 0.001 & 0.121 & 0.007 & +0.013 \\
\hline $\begin{array}{l}\text { Polarity } \\
(-1: 1)\end{array}$ & -0.193 & 0.002 & -0.160 & 0.004 & -0.034 \\
\hline No of Pronouns & 3.553 & 0.012 & 3.565 & 0.075 & -0.012 \\
\hline Readability (0-100) & 61.933 & 0.085 & 63.61 & 0.491 & -1.680 \\
\hline
\end{tabular}

Difference between early and late posts

On Gab, users' initial hate messages differed both in style and substance from later messages (Table 2). Early messages had higher levels of obscenity, more explicit and severe hate terms, lower lexical density, a higher level of 'othering', and held a more negative sentiment. These messages also contained fewer hate symbols. In other words, early hate posts seemed more explicit and less nuanced. They also received more reactions from other users than later hate posts. Initial and later hate posts were posted on days with similar overall hate level on the platform.

Users also increased the breadth of their hate targets over time from early to later hate messages. Within users' initial 100 messages, on average they expressed hate towards $3.2+/-0.01$ target groups, and this increased to $5.2+/-0.02$ for the subsequent 100 messages. By comparison, an average user's initial ten messages contained hate messages targeted towards $1.6+/-0.001$ different groups.

\section{Engagement with hate messages}

The level of engagement a post received varied depending on the level of abuse it contained. 'Hate speech' messages received the greatest engagement, followed by 'offensive' then 'clean' messages (Mean normalised reactions: clean $=0.88+/-0.0002$, offensive $=0.90+/-0.0005$, hate speech $=1.1$ $+/-0.0005$, Figure 5a). The level of engagement a hate post received also marginally increased the likelihood of the user posting subsequent hate messages. Users who received no reactions to their hate 


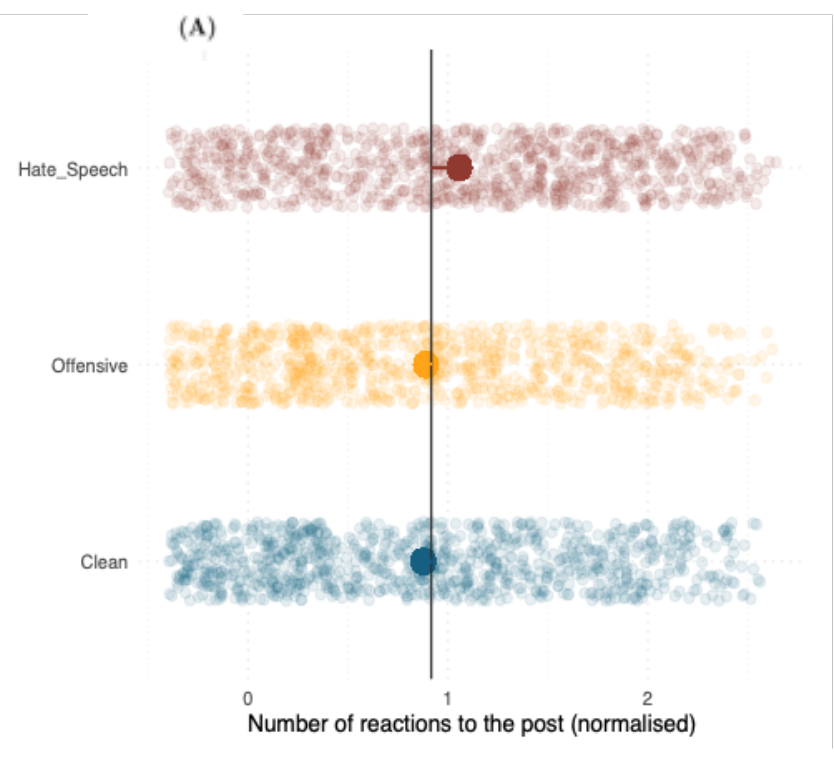

(B)

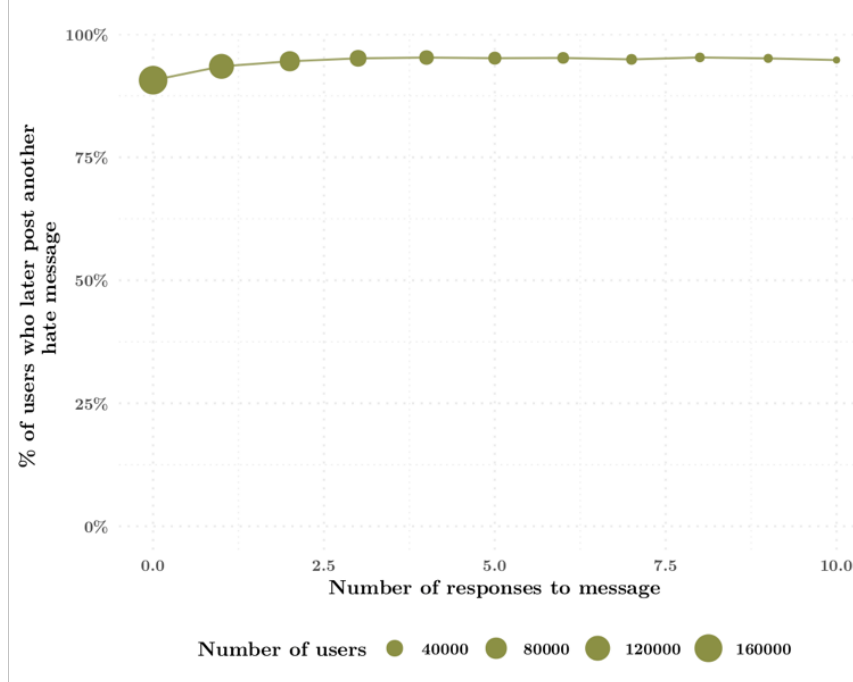

Figure 5 - A) Number of reactions to clean, offensive and hate speech messages on Gab.

Circles show mean number of reactions per type, the black line show mean reactions across all types.

$B)$ Probability that a hate speech message will be followed by another for varying levels of reactions

messages had a $90.8 \%$ likelihood of posting another hate message, and this increased to $95.2 \%$ when a hate post received 10 reactions (Figure $5 \mathrm{~b}$ ).

\section{Social contagion of online hate speech}

We observed a strong overall effect of social contagion of hate speech at the short-term (hourly) and longer-term (daily/weekly) levels.

At the short-term (hourly) level, exposure to hate speech in a given hour increased the likelihood that a user would express hate speech themselves in the following hour (GLMM, $\mathrm{n}=11,034,480$, Estimate $=0.61 \pm 0.08$, Wald's Z $=7.73, \mathrm{p}<0.001$, Figure 6a, SI Figure 8\&9). Each $10 \%$ increase in exposure to hate speech increased the odds of a user expressing hate themselves in the subsequent hour by $8.5 \%$, while a user with $100 \%$ of their network connections expressing hate speech was $85 \%$ more likely to adopt this behaviour than a user with zero connections expressing hate speech.

To test for longer-term social contagion effects of exposure to hate speech we measured a user's total daily exposure to hate and their average exposure over the prior seven days. Results showed that both of these types of exposure influenced future hate expression (Figure 7a\&b, GLMM, $\mathrm{n}=479,760$, Daily exposure: Estimate $=1.30 \pm 0.47$, Wald's $\mathrm{Z}=2.76, \mathrm{p}=0.006$. Weekly exposure: Estimate $=1.30 \pm$ 0.47 , Wald's $\mathrm{Z}=2.76, \mathrm{p}=0.006$ ). Comparing these two models, results indicate that longer-term exposure to hate speech over the previous seven days had a larger impact on whether a user expressed hate speech themselves compared to daily exposure ( $\Delta$ AIC between the two GLMMs $=-74.5$ ).

Interestingly, within these models, the number of days a user had been active prior to the focal day was also a significant predictor of hate speech expression, even when accounting for prior levels of exposure to hate speech in both the daily and weekly models. (GLMM, Weekly Model; Days active: Estimate $=0.002 \pm 0.001$, Wald's $\mathrm{Z}=2.26, \mathrm{p}=0.024$. Daily Model; Days active: Estimate $=0.002 \pm$ 0.001 , Wald's $\mathrm{Z}=2.06, \mathrm{p}=0.039$ ) 
Transfer effects of exposure to hate speech

We tested whether this social contagion effect was specific to the type of hate speech exposed to, or whether there were secondary transfer effects across hate types. At the short-term (hourly) level, results indicated that while the strongest effects were for matching hate targets, there were also significant transitive effects across hate types, i.e., users exposed to one type of hate speech then went on to express another type of hate, at a rate above chance (Figure 6b, Table 3 ). This was particularly pronounced for users exposed to anti-Semitism, Islamophobia, and anti-Black racism. The effects of this transfer of hate appear strongest between targeted groups which have overlapping traits, such as Islamophobia and anti-immigration. Many combinations of hate targets did not transfer however (Table 3), suggesting that there is also a degree of specificity to these effects.

We found similar patterns at the daily/weekly (Table 4), with anti-Semitism and Islamophobia having the greatest transitive effects, followed by anti-immigration sentiment. The effect of exposure to antiBlack racism, misogyny and homophobia appeared less transferable and therefore more specialised. (See SI Table 2 for short-term transfer effects when controlling for these long-term trends).

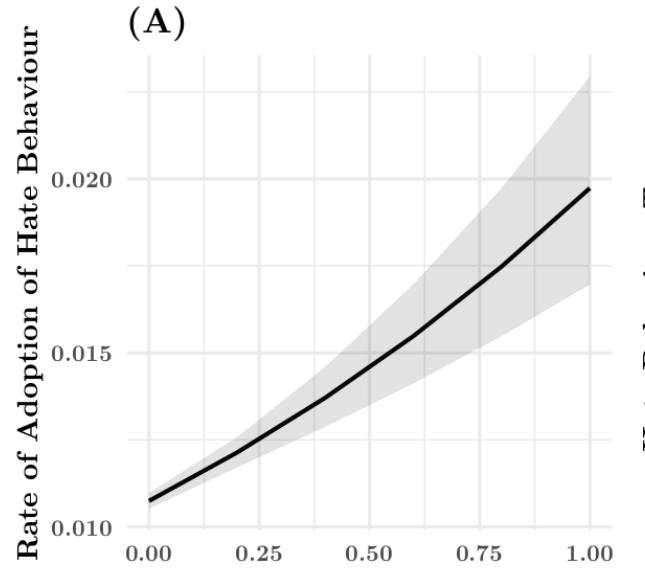

Overall Hate Speech Exposure (Hourly)
(B)

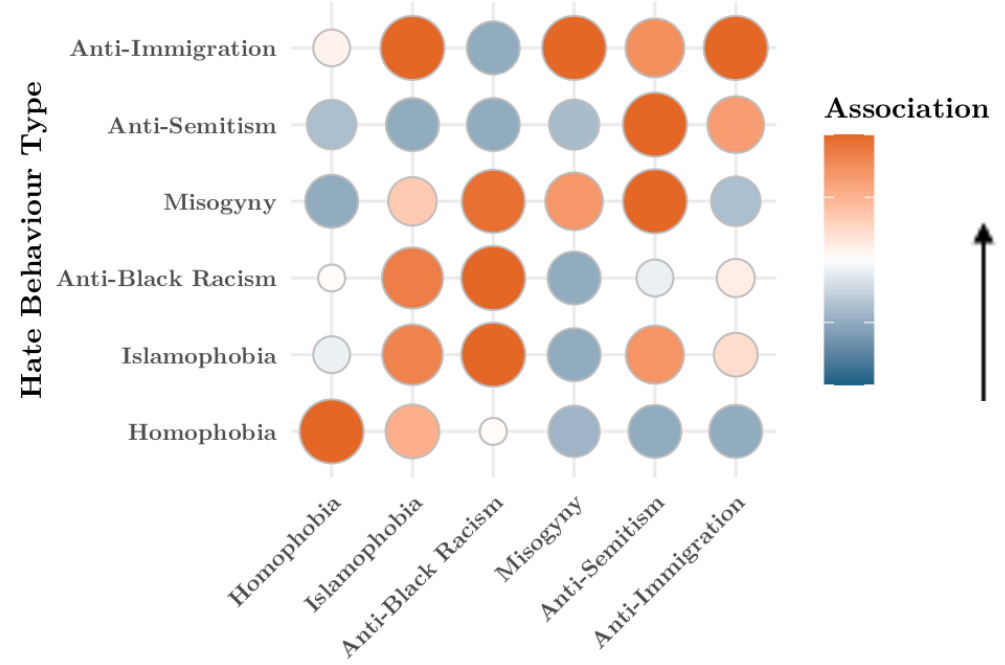

Hate Exposure Type

Figure 6 - A) Overall effect of exposure to hate speech on adoption of this behaviour (the grey area represents the 95\% CI, the large CI towards high hate speech exposure is caused by low sample sizes of users exposed to such levels of hate). B) Effect sizes for exposure to hate types on adoption of these hate behaviours. Colour and point size indicate strength of association 
Table 3 - Statistical effects of exposure to hate types on the likelihood to express hate speech of this or another type (short-term model). The blue bars show the relative proportion of each hate type. Significant relationships are highlighted

$$
\text { in yellow. }
$$

Hate Speech Exposure Type

\begin{tabular}{|c|c|c|c|c|c|c|c|c|c|c|c|c|c|c|c|c|c|c|c|}
\hline \multirow{2}{*}{$\begin{array}{l}\text { OBSERVED } \\
\text { BEHAVIOUR }\end{array}$} & \multicolumn{3}{|c|}{ Anti-semitism } & \multicolumn{3}{|c|}{ Islamophobia } & \multicolumn{3}{|c|}{ Misogyny } & \multicolumn{3}{|c|}{ Anti Black Racism } & \multicolumn{3}{|c|}{ Anti Immigration } & \multicolumn{3}{|c|}{ Homophobia } & \multirow{2}{*}{$\begin{array}{c}\text { RELATIVE } \\
\text { PROPROTION }\end{array}$} \\
\hline & ESTIMATE & F & $\mathbf{P}$ & ESTIMATE & F & $P$ & ESTIMATE & F & $\mathbf{P}$ & ESTIMATE & F & $\mathbf{P}$ & ESTIMATE & F & $\mathbf{P}$ & ESTIMATE & F & $\mathbf{P}$ & \\
\hline Anti-Semitism & 1.9 & 11.4 & 0.000 & 1.2 & 6.9 & 0.000 & 1.1 & 2.1 & 0.168 & 1.0 & 1.9 & 0.056 & 1.7 & 8.0 & 0.000 & 1.1 & 2.133 & 0.165 & \\
\hline Islamophobia & 1.3 & 7.5 & 0.000 & 1.2 & 9.5 & 0.000 & 0.1 & 0.2 & 1.000 & 1.6 & 4.4 & 0.000 & 0.8 & 3.5 & 0.002 & 0.5 & 1.039 & 1.000 & \\
\hline Misogyny & 2.2 & 6.5 & 0.000 & 1.3 & 3.3 & 0.001 & 1.8 & 1.7 & 0.354 & 2.2 & 2.5 & 0.042 & 0.4 & 0.4 & 0.675 & 0.1 & -0.002 & 1.000 & \\
\hline $\begin{array}{l}\text { Anti-Black } \\
\text { Racism }\end{array}$ & 0.7 & 1.0 & 0.309 & 1.7 & 5.4 & 0.000 & 0.1 & -0.0 & 1.000 & 2.2 & 2.6 & 0.034 & 1.0 & 1.5 & 0.396 & 0.8 & 0.560 & 1.000 & \\
\hline $\begin{array}{c}\text { Anti- } \\
\text { immigration }\end{array}$ & 1.4 & 7.5 & 0.000 & 1.5 & 9.4 & 0.000 & 1.9 & 6.0 & 0.000 & -1.4 & -3.4 & 0.004 & 1.9 & 8.6 & 0.000 & -0.0 & -0.134 & 1.000 & \\
\hline Homophobia & 0.9 & 1.6 & 0.233 & 2.0 & 6.6 & 0.000 & 0.9 & 1.3 & 0.625 & 1.5 & 2.2 & 0.054 & 1.0 & 1.4 & 0.396 & 2.7 & 4.657 & 0.000 & \\
\hline
\end{tabular}


(A)

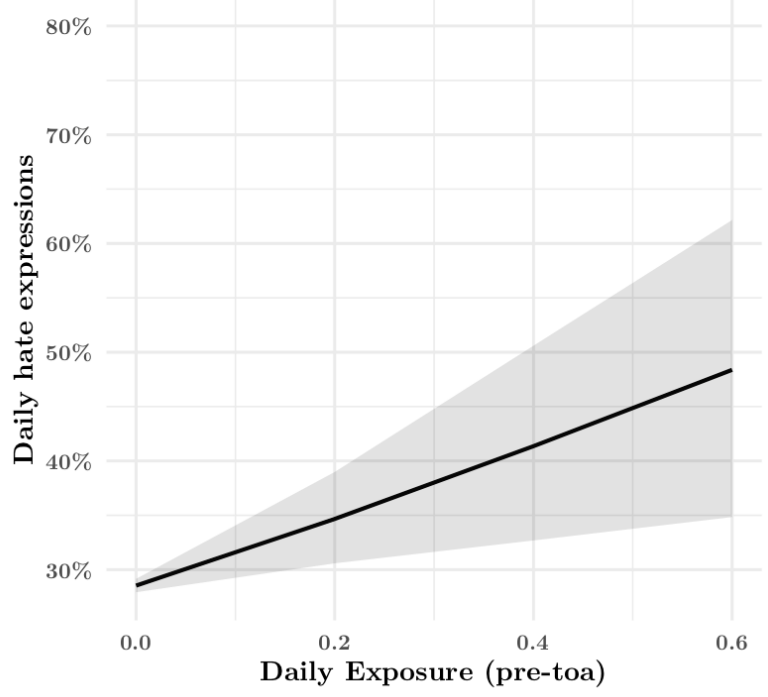

(B)

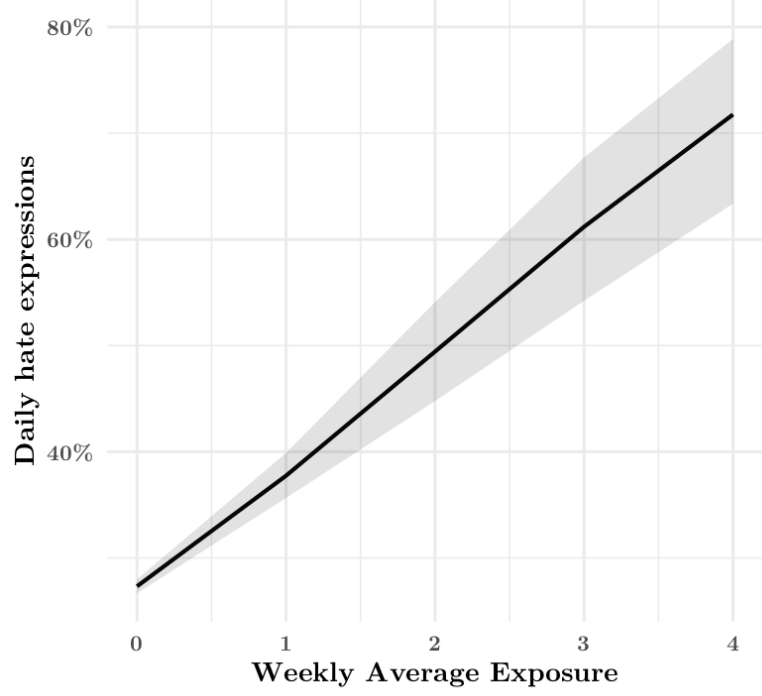

Figure 7 - A) Effect of short-term exposure to hate types on adoption of these hate behaviours while controlling for longterm exposure. B) Long-term effect of exposure to hate speech on adoption of this behaviour controlling for short-term exposure.

Table 4 -Statistical effects of 7-Day exposure to hate types on the likelihood to express hate speech of this or another type (longer-term model). Significant relationships are highlighted in yellow.

Hate Speech Exposure Type

\begin{tabular}{|c|c|c|c|c|c|c|c|c|c|c|c|c|c|c|c|c|c|c|}
\hline \multirow{2}{*}{$\begin{array}{l}\text { BEHAVIOUR } \\
\text { (HATE TYPE) }\end{array}$} & \multicolumn{3}{|c|}{ Anti-Semitism } & \multicolumn{3}{|c|}{ Islamophobia } & \multicolumn{3}{|c|}{ Misogyny } & \multicolumn{3}{|c|}{ Anti Black Racism } & \multicolumn{3}{|c|}{ Anti Immigration } & \multicolumn{3}{|c|}{ Homophobia } \\
\hline & ESTIMATE & F & $P$ & ESTIMATE & F & $P$ & ESTIMATE & F & $P$ & ESTIMATE & F & $P$ & ESTIMATE & F & $P$ & ESTIMATE & F & $P$ \\
\hline \multicolumn{19}{|l|}{7 Day Average } \\
\hline Anti-Semitism & 1.30 & 8.00 & 0.00 & 0.71 & 5.86 & 0.00 & 0.26 & 0.50 & 1.00 & -0.09 & -0.06 & 1.00 & 0.55 & 2.91 & 0.03 & 0.75 & 0.41 & 1.00 \\
\hline Islamophobia & 0.71 & 5.63 & 0.00 & 0.86 & 9.38 & 0.00 & 0.45 & 1.28 & 0.40 & 0.60 & 1.95 & 0.20 & 0.76 & 5.30 & 0.00 & 1.35 & 4.20 & 0.00 \\
\hline Misogyny & 0.40 & 1.08 & 1.00 & 0.41 & 1.42 & 0.94 & 1.17 & 1.14 & 1.00 & -1.75 & -1.18 & 1.00 & 0.91 & 1.69 & 0.83 & 1.31 & 0.96 & 1.00 \\
\hline $\begin{array}{l}\text { Anti-Black } \\
\text { Racism }\end{array}$ & 0.14 & 0.33 & 1.00 & 0.10 & 0.36 & 1.00 & 0.59 & 0.62 & 1.00 & 3.23 & 5.65 & 0.00 & 0.38 & 0.80 & 1.00 & -2.82 & -1.75 & 0.72 \\
\hline $\begin{array}{c}\text { Anti- } \\
\text { Immigration }\end{array}$ & 0.61 & 3.36 & 0.01 & 0.56 & 4.38 & 0.00 & 0.28 & 0.52 & 1.00 & -0.10 & -0.16 & 1.00 & 0.50 & 2.39 & 0.13 & 0.65 & 1.22 & 1.00 \\
\hline Homophobia & 1.07 & 2.42 & 0.15 & 0.18 & 0.58 & 1.00 & -0.24 & -0.20 & 1.00 & -3.56 & -1.95 & 0.41 & 0.62 & 1.26 & 1.00 & -0.05 & -0.05 & 1.00 \\
\hline
\end{tabular}

\section{Discussion}

This study investigates how hate develops on fringe social media platforms and how user's hate speech expression relates to their participation in the social media platform and to the exposure to hate from they received from other users. We found that hate speech expressions increased with time spent on the platform, although a number of users started posting hate from their first post, and that higher exposure to hate speech increased the likelihood that users expressed hate speech themselves, over both short and longer timeframes. This effect was largest for the specific type of hate that users have been exposed to, but also transitive across hate types. Overall, our results (i) provide evidence socialisation effects on hate speech expression on social media platforms, without excluding that some users join platforms with pre-existing prejudice, (ii) reveal that hate speech on social media platform 
spreads through social contagion, both in the short and longer term, and (iii) highlight the existence of transfer effects across hate types following exposure to hate speech from other ingroup members.

We found a substantial quantity of hate speech on Gab, in line with previous research (Mathew et al., 2019; Zannettou et al., 2018), which highlights the damage these spaces may do to group relations and the promotion of violence (Tajfel, 1974; Williams, Burnap, Javed, Liu, \& Ozalp, 2019). In agreement with previous research on other fringe platforms (Finkelstein, Zannettou, Bradlyn, \& Blackburn, 2020; Kleinberg et al., 2020; Ribeiro et al., 2020), we also find that the overall density of hate speech on Gab has increased over time, stressing the importance on understanding the impact that this hate has on users and the wider community.

\section{Socialisation effects on hate speech expression}

At the platform level, we found evidence that socialisation affects hate expression through interactions with other group members. The proportion of a user's posts containing hate speech increased with the number of posts they contributed to the platform. This relationship was also true for the number of days users spent on the platform regardless of their posting activity, suggesting an effect of observation (or 'lurking') on driving hate speech expression even without active participation in the conversations. This supports suggestions that fringe platforms such as Gab play a role in connecting more moderate right-leaning users to extreme far-right ideologies (McSwiney \& Jasser, 2020).

Investigating hate trajectories at the user level allowed us to better understand how platform usage affects hate speech expression, and to test whether users are drawn to these platforms to express preexisting hate, or whether this hate develops with presence on the platforms. We find evidence for both effects playing a role. There was a consistent 'U-shaped' pattern in users' hate trajectories, indicating that the likelihood of hate expression was high for the first few messages, before decreasing then rising again as users spent longer on the platform. Like our platform-wide results, this latter increase suggests that users become more extreme in their expressions as they spend longer on the platform, taking on the group norms through a process of socialisation (Smith et al., 2019). This supports evidence that social media use is associated with outgroup prejudice and affective polarisation between mainstream groups (Settle, 2018), and goes a step further by showing that this may also hold for extreme negative and hostile outgroup attitudes. The upwards trajectory in hate speech expression occurred even when taking into account the overall density of hate messages posted on the days the users were active, suggesting that this effect is not simply driven by immediate group conversations or topics, but by a deeper internalisation of the hostile sentiment. Overall, this supports our hypothesis that users who spend time on hateful fringe social media platforms increase the hateful nature of their own messages, likely through socialisation effects.

To further investigate these apparent socialisation effects on hate speech expression, we looked at the number of responses, and therefore engagement / attention, that different types of message received. Hate speech messages received the greatest engagement, compared to offensive or clean message. This aligns with previous research showing that participating in online hate can increase a user's online visibility and subsequently increases their influence on the platform as a whole (ElSherief, Nilizadeh, Nguyen, Vigna, \& Belding, 2018). This result also supports the idea that hate speech occurs through group socialisation, because over time influential users will start to shape not just their own expressions but also drive the extremity of the group as a whole, which leads to a reciprocal relationship between the individual's and the group's extremity (Levine \& Moreland, 1994). We did not find a large effect of engagement to hate speech encouraging the users to post hate again, and the likelihood of posting hate messages remained fairly constant regardless of reactions, but this may have 
been due to a ceiling effect caused by the long 2-year period of observation with most hate messages followed by another regardless of social feedback over this period.

\section{Evidence for pre-existing prejudice}

The high starting position and initial drop in hate speech expression we found in users' hate speech trajectories points towards another effect for early hate postings however, and suggests that socialisation with other users may not be the sole cause of hate expression. As such, there may be differential explanations and causes for early vs later hate speech expressions. This is supported by the fact that the first hate message on Gab was posted after just 46 other posts and 1.5 days after the platform was created. These early hate posts are very unlikely to result from group socialisation and therefore indicate pre-established hate must have pre-dated the platform.

There are three possible causes for these high early hate levels. Firstly, users may deliberately join these platforms to express hate speech and seek out similar extreme content for themselves (Pauwels \& Schils, 2016). Alternatively, users may over-express hate speech in their initial posts to 'prove' group membership, loyalty, and to gain acceptance by other in-group members (Doosje et al., 2016; Harel, Jameson, \& Maoz, 2020). Offline evidence suggests that peripheral members of a desirable ingroup derogate an outgroup in public to enhance status, even if they do not hold this attitude in private (Noel, Wann, \& Branscombe, 1995). Finally, there may be a period of 'observation' or 'lurking' before users post a first message, during which they may build up an affective state in relation to the outgroup through the influence of the emotional expressions of others, and only post a message when this reaches a certain threshold (Brady, Wills, Jost, Tucker, \& Van Bavel, 2017a; Van Kleef, 2009; Van Kleef, Van Den Berg, \& Heerdink, 2015).

In the current study it is not possible to definitively test for which of these explanations is likely to be correct. However, several of our findings can help further indicate that users' initial hate posts may be driven by pre-existing opinions which they have come to the platform to present, but over time the nature of their hate expressions both increases and evolves to adopt the norms of the online community as a whole. Users' initial hate posts were more explicit and less nuanced compared to later posts; they contained a higher proportion of explicit hate terms, high obscenity, lower sentiment, and fewer obfuscated hate symbols. This lack of subtlety, together with the fact that ingroup specific language only occurred in later hate posts, suggest that the latter develops by spending time on the platform, supporting the idea that differential causes underpin early and late hate postings (Tuters \& Hagen, 2019). Similarly, the number of outgroups that users targeted with hate increased over time spent in the platform, while users initial hate targets were more focused on a specific group. This supports ideas that prejudice or 'fixation' against a certain group may drive users onto these platforms (Cohen, Johansson, Kaati, \& Mork, 2014; Grover \& Mark, 2019), but this prejudice may expand to other groups through interactions on the platform. Finally, the hate trajectories of the most active users did not show this u-shaped trajectory, and instead we observed a more consistent overall upwards pattern in hate speech expressions. This indicates that the initial drop may be driven by users who do not remain active in the platform over the longer term, suggesting that this drop might be caused by 'venting behaviour' after which the users are satiated and leave the conversation. Similar patterns, with many users quickly leaving the platform, have been observed on far-right extremist forums (Scrivens, Wojciechowski, \& Frank, 2020). The overall hate speech on the platform was not higher for users' initial hate post, suggesting that affective triggers or 'outrage' (Brady et al., 2020; Brady, Wills, Jost, Tucker, \& Van Bavel, 2017) may not be solely driving initial hate posts-users are less likely to have seen something which makes them respond with hostility if the overall platform is no more hate-filled. 


\section{Social contagion effects on hate speech expression}

Another key finding of this study is that social contagion appears to play a role in hate speech expression. Indeed, users who experienced greater exposure to hate speech from other users they interacted with on the platform were more likely to subsequently express hate speech themselves. Our results support prior work which shows that emotional states can spread between users on social media (Bond et al., 2012; Brady et al., 2017b; Kramer et al., 2014; Terizi et al., 2020), and demonstrate that such effects are also applicable to more extreme prejudicial behaviours. This raises concerns about the negative impacts of the online environment on intergroup relations and conflict.

This effect of exposure on hate expressions occurred in both the short- (hourly) and longer-term (daily/weekly) but was larger following longer-term exposure, suggesting that these effects may accumulate over time and reflect longer term changes in attitude rather than reactionary responses to the immediate conversations that users are a part of. Interestingly, in these longer-term models we observed that the total duration of user activity on the platform increased the likelihood of expressing hate speech, regardless of the exposure patterns they experienced. This is in accordance with our earlier results that suggest time spend on the platform (in days) was correlated with proportion of hate speech messages, and suggests an effect of passive consumption of hate, in addition to the measured activity of interactions with other users. This supports prior evidence that within social media users are influenced as much by the content that they passively consume as that which they interact with (Settle, 2018), and that these even users who never contribute to conversations consider themselves a member of the online community (Nonnecke, 2000). It is not possible for us to measure this passive consumption from publicly available data, but in future exploring this may give valuable insight into how hate speech spreads online even amongst those active but not participatory.

The social contagion effect of hate speech we observe suggests that conformity effects are influencing users' propensity to express hate speech themselves. In other words, if a greater proportion of their direct connections are themselves expressing hate, then they themselves are also more likely to do so. This supports traditional ideas about the role of conformity in prejudice (Allport, 1954). The Internet, and social media in particular, has been implicated in skewing conformity pressures by giving the impression of false consensus on extreme positions (Pariser, 2011). Those with the most extreme ideological positions are the most active (Barberá \& Rivero, 2015; Preotiuc-Pietro, Liu, Hopkins, \& Ungar, 2017), receive the most engagement (as discussed above), and this may be accentuated on fringe platforms due to self-selection bias (Robbinson, Schlegel, Janin, \& Deverell, 2020). Together, this means that conformity along the lines of the majority behaviour of social media connections may be driving users to more extreme online social norms than exist in reality. This is likely to play a role in exacerbating intergroup conflict as groups adopt increasingly extreme positions (Reicher et al., 2008; Smith et al., 2019). Conformity effects are especially pronounced in far-right groups and in those scoring higher in right-wing authoritarianism (Altemeyer, 1981), which can result in the acceptance of political ideologies that demean outgroups. In addition, influential 'extremist' leaders who deviate from the average group position have a disproportionate impact on the overall group position (Sherif, 1936). Given that in online environments these leaders will form central nodes in the networks, they will also be responsible for much of the hate speech exposure patterns (Barberá et al., 2015; Susarla, Oh, \& Tan, 2012), and therefore adoption patterns, of other users (Haslam, Reicher, \& Bavel, 2019).

\section{Transfer effects for hate contagion}

Another important result of our study relates to the transitive effects of hate speech contagion. Users were most likely to express the same type of hate to which they had been exposed, which supports evidence that group members learn their negative outgroup attitudes from other ingroup members (Bracegirdle, 2020). However, we also found substantial transfer effects, at both the short-term hourly 
conversation level, and the longer-term daily/weekly level. This indicates that group members' negative outgroup attitudes may propagate across outgroups in a similar fashion to the transfer of positive attitudes in cases of positive intergroup contact (Pettigrew, 2009; Schmid et al., 2014; Tausch et al., 2010). This has important implications for group extremism and intergroup conflict, because as the number of outgroups targeted with hate increases, the negative contact opportunities increase and the likelihood of resolution decreases. There is evidence that exposure to hate speech online can lead to an effect of 'nebulous othering', whereby groups construct an increasingly vaguely defined 'other' to whom prejudice and intolerance is targeted (Tuters \& Hagen, 2019). Ultimately, this transfer effect of hate speech across groups could expand to encompass all those not part of the ingroup.

Transfer effects were largest for types of hate which could be viewed as similar (Islamophobia and anti-immigration sentiment), but also crossed what might be seen as wider group boundaries (homophobia and Islamophobia / misogyny and anti-immigration for example). This may be in part due to the way that certain hate types are presented. For instance, much anti-immigration sentiment is presented by the far-right as being in defence of women and preventing foreign 'defilement' of Western women by 'invaders' (Davey \& Ebner, 2017; Ekman, 2019; Olteanu, Castillo, Boy, \& Varshney, 2018). Misogyny could then easily derive from this stance (Sarrasin, Fasel, Green, \& Helbling, 2015). This combination and conflation of hate targets may partly explain the transfer of hate from one target group to another. However, our approach precludes us from exploring this because we assigns one type of hate to each hate message. Future research should explore this phenomenon in greater detail by including more detailed and nuanced hate type distinctions and investigate whether this is a true transfer across distinct outgroups or instead a 'step up' in abstraction where the outgroup encompasses wider distinctions (all foreigners for example).

\section{Limitations and future research}

When discussing the propensity to for users to express hate on social media is important to acknowledge that the majority of users do not exhibit this behaviour, even on fringe platforms. For example, in this study $71.1 \%$ of users never expressed hate speech. The reasons for this are unclear, and we did not focus on these users, however further investigating these non-hateful users may be vital for discovering and developing mitigation strategies for the spread of hate speech online. Similarly, in this work we rely on the measurement of interactions with the platform, which leaves a digital trace, rather than consumption of content which does not. Users are influenced as much by the content that they passively consume (Settle, 2018), and while recent work has made progress in building estimates of users' consumption behaviours on Twitter (Dunn et al., 2020), this needs validating and expanding to other social media platforms. Future work should investigate how much of user's outgroup attitudes can be affected by the content which they passively consume from other ingroup members, compared to active interactions. This is especially important given that the production of content is highly unequal, with a few users creating most of the content (Nielsen, 2006). This is also true for hate speech on these fringe platforms (SI Figure 7), and future work should therefore look to explore the impact on this majority of users who consume social media content rather than create it.

In this study we have also made a number of assumptions in the creation of the contagion networks which should be tested further in future work. Notably, while we have endeavoured to measure 'exposure' to online hate speech through network connections, we cannot be sure that this network measure relates directly to the content that users actually see on screen or attend to if it is presented. The users may simply not be online when their network connections post hate, or they may not read it even if they are online, however it is not possible to measure this from social media trace data alone. In aggregate we can assume a positive relationship between the rate at which connections post 
hate and the amount of hate users sees when active on the platform, but for individual users this may not hold. Future work should investigate this relationship between network exposure and the level of hate speech exposure experienced. Additionally, in order to implement the diffusion network models we made the assumption that once an individual has adopted the behaviour they remain in this state, and do not reverse or indeed get 'infected' again. This is an oversimplification of real-world behaviours, and in reality, users can express hate speech multiple times, and indeed change between hateful and tolerant states quickly and easily (Rajadesingan et al., 2020). We address this, in part, by looking at multiple days and allowing users to adopt different positions on different days- but differential effects may still occur for multiple 'adoptions' of hate behaviour within a single day. These assumptions, and the effect of user's multiple adoptions of hate or indeed retractions of hate on the behaviour of those around them, should be tested. In a similar way, the relationship between exposure and likelihood of adoption of behaviour within online social networks is likely to follow a complex rather than simple relationship; users adoption patterns will depend on the numbers of adopters within a user's social vicinity, with the adoption probability increasing slowly for low numbers of unique exposure sources, and then quickly when the number of exposure sources reaches a threshold level (Mønsted, Sapieżyński, Ferrara, \& Lehmann, 2017). This means that non-linear adoption patterns may occur. Future work should investigate this threshold effect and identify if thresholds are constant across users. If so, then mitigation strategies which can ensure that exposure remains below this threshold might be a useful 'soft' mitigation strategy.

Future research should also make attempts to analyse multiple social media platforms and consider the interplay between platforms and in particular the changes caused by moderation efforts and bans from mainstream platforms. Hate speech is not restricted to a single social media platform or online group and the hate ecosystem is multi-dimensional, and these wider effects should be taken into account (Johnson et al., 2019). This is especially important as mainstream social media platforms enforce more stringent content moderation policies and users migrate to smaller platforms where exposure patterns to hate speech may be more concentrated (Urman \& Katz, 2020). Additionally, in this work we also do not distinguish group membership of those posting hate speech, and therefore there is a chance that some of the hate is directed from out-group members at the platform or ingroup itself. In this way some initial hate posts could be a 'trolling effect' where users turn up to post hate 'about' the group and then leave (Graham, 2019; Honeycutt, 2006). Future work should consider both intergroup and intragroup communication effects on hate speech expression.

Finally, an important aspect to consider in future is the relationship between online hate speech to the offline world. It is unlikely that the Internet alone is the cause of extremism, intergroup conflict, and hate (Meleagrou-Hitchens \& Kaderbhai, 2016), and offline relationships and events are key in the development of extreme opinions and behaviours (Koehler, 2014). There is evidence that online hate speech can spike in the days and weeks following offline violence such as terror attacks and mass shootings (Rowe \& Saif, 2016; Theocharis et al., 2019; Zannettou et al., 2020; Burnap \& Williams 2014; Williams \& Burnap, 2016). How hate speech changes following such events, whether it is specific to the type of event (e.g. Islamophobia following a Islamic terror attack) or more generalised, and whether the relationship also holds in reverse where hate speech against a certain group increases prior to offline violence against this group, should be investigated. This is particularly important given evidence that interactions between ingroup members can invoke radical intentions if external prompts for legitimising beliefs are present (Thomas et al., 2014). In this way, offline violence such as terror attacks may act as the legitimising external prompt, leading to a shared group belief in the perceived grievance and an increase in hate speech towards a target group. This should be tested in future work. 


\section{Conclusion}

This work provides new insight into the drivers of hate speech expression online, highlighting the role of group socialisation and social contagion. By participating in the conversations on fringe social media platforms popular with the far-right, individuals become increasingly likely to express hate themselves. This effect of exposure to hate speech is not limited to one type of hate and appears to transfer across hate targets. These results are likely widely applicable to other platforms and types of extremism, and have important implications for our understanding of hostile intergroup relations and the role of the Internet in intergroup conflict and hate. As fringe social media platforms become more popular with far-right extremist groups, these patterns of hate exposure will increase, potentially driving dangerous trends in hate towards outgroups. 


\section{References}

Agarwal, S., \& Sureka, A. (2015). Applying social media intelligence for predicting and identifying on-line radicalization and civil unrest oriented threats. ArXiv, 1-18. Retrieved from http://arxiv.org/abs/1511.06858

All-Party Parliamentary Group (APPG) on Hate Crime. (2019). How do we build community cohesion when hate crime is on the rise? Retrieved from www.appghatecrime.org

Allport, G. (1954). The nature of prejudice. Addison-Wesley Publishing Company.

Alorainy, W., Burnap, P., Liu, H., \& Williams, M. (2018). The enemy among us: Detecting hate speech with threats based "othering" language embeddings. ACM Transactions on the Web, 9(4), 1-26. Retrieved from http://arxiv.org/abs/1801.07495

Altemeyer, B. (1981). Right-wing authoritarianism. University of Manitoba Press.

Anoop, V. S., Asharaf, S., \& Deepak, P. (2016). Unsupervised concept hierarchy learning: A topic modeling guided approach. Procedia Computer Science, 89, 386-394. https://doi.org/10.1016/j.procs.2016.06.086

Anti-Defamation League. (2019). When Twitter bans extremists, GAB puts out the welcome mat. Retrieved March 20, 2019, from https://www.adl.org/blog/when-twitter-bans-extremists-gab-puts-out-the-welcomemat

Arun, R., Suresh, V., Madhavan, C. E. V., \& Murthy, M. N. N. (2010). On finding the natural number of topics with latent dirichlet allocation: Some observations. Advances in Knowledge Discovery and Data Mining, 1138. Retrieved from http://hdl.handle.net/10722/26088

Asch, S. (1955). Opinions and social pressure. Scientific American, 193(5), 31-35. https://doi.org/10.1038/scientificamerican1155-31

Bakshy, E., Rosenn, I., Marlow, C., \& Adamic, L. (2012). The role of social networks in information diffusion. WWW'12 - Proceedings of the 21st Annual Conference on World Wide Web, 519-528. https://doi.org/10.1145/2187836.2187907

Barberá, P. (2015). Birds of the same feather tweet together: Bayesian ideal point estimation using twitter data. Political Analysis, 23(1), 76-91. https://doi.org/10.1093/pan/mpu011

Barberá, P., \& Rivero, G. (2015). Understanding the political representativeness of Twitter users. Social Science Computer Review, 33(6), 712-729. https://doi.org/10.1177/0894439314558836

Barberá, P., Wang, N., Bonneau, R., Jost, J. T., Nagler, J., Tucker, J., \& González-Bailón, S. (2015). The critical periphery in the growth of social protests. PLoS ONE, 10(11), 1-15. https://doi.org/10.1371/journal.pone.0143611

Benson, T. (2016). Inside the "Twitter for racists": Gab — the site where Milo Yiannopoulos goes to troll now. Salon. Retrieved from https://www.salon.com/2016/11/05/inside-the-twitter-for-racists-gab-the-site-wheremilo-yiannopoulos-goes-to-troll-now/

Berger, J. M. (2018). Extremism. Cambridge, Massachusetts: The MIT Press.

Blei, D., Ng, A., \& Jordan, M. (2003). Latent Dirichlet Allocation. Journal of Machine Learning Research, 12(4), 421. https://doi.org/10.2307/3050792

Bond, R. M., Fariss, C. J., Jones, J. J., Kramer, A. D. I., Marlow, C., Settle, J. E., \& Fowler, J. H. (2012). A 61million-person experiment in social influence and political mobilization. Nature, 489(7415), 295-298. https://doi.org/10.1038/nature11421

Bracegirdle, C. (2020). Exploring the limits of intergroup contact: Ingroup contact and group status. University of Oxford.

Brady, W. J., Crockett, M. J., \& Van Bavel, J. J. (2020). The MAD model of moral contagion: The role of motivation, attention, and design in the spread of moralized content online. Perspectives on Psychological Science, 15(4), 978-1010. https://doi.org/10.1177/1745691620917336

Brady, W. J., Wills, J. A., Jost, J. T., Tucker, J. A., \& Van Bavel, J. J. (2017a). Emotion shapes the diffusion of moralized content in social networks. Proceedings of the National Academy of Sciences, 114(28), 73137318. https://doi.org/10.1073/pnas.1618923114

Brady, W. J., Wills, J. A., Jost, J. T., Tucker, J. A., \& Van Bavel, J. J. (2017b). Emotion shapes the diffusion of moralized content in social networks. Proceedings of the National Academy of Sciences, 114(28), 73137318. https://doi.org/10.1073/pnas.1618923114

Braun, A. F., Dickey, B., Heide, J. Van Der, Maragkou, E., Peeters, S., \& Steffen, B. (2020). Normiefication of extreme speech and the widening of the Overton window. Retrieved from https://oilab.eu/normiefication-ofextreme-speech-and-the-widening-of-the-overton-window/ 
Brown, R., \& Hewstone, M. (2005). An integrative theory of intergroup contact. Advances in Experimental Social Psychology, 37, 255-343. https://doi.org/10.1016/S0065-2601(05)37005-5

Burnap, P., \& Williams, M. L. (2015). Cyber hate speech on twitter: An application of machine classification and statistical modeling for policy and decision making. Policy and Internet, 7(2), 223-242. https://doi.org/10.1002/poi3.85

Burnap, P., Williams, M. L., Sloan, L., Rana, O., Housley, W., Edwards, A., ... Voss, A. (2014). Tweeting the terror: modelling the social media reaction to the Woolwich terrorist attack. Social Network Analysis and Mining, 4(1), 1-14. https://doi.org/10.1007/s13278-014-0206-4

Cao, J., Xia, T., Li, J., Zhang, Y., \& Tang, S. (2009). A density-based method for adaptive LDA model selection. Neurocomputing, 72(7-9), 1775-1781. https://doi.org/10.1016/j.neucom.2008.06.011

Centola, D. (2010). The spread of behavior in an online social network experiment. Science, 329(5996), 11941197. https://doi.org/10.1126/science.1185231

Chandrasekharan, E., Jhaver, S., Bruckman, A., \& Gilbert, E. (2020). Quarantined! Examining the effects of a community-wide moderation intervention on Reddit. ArXiv. Retrieved from http://arxiv.org/abs/2009.11483

Chandrasekharan, E., Pavalanathan, U., Srinivasan, A., Glynn, A., Eisenstein, J., \& Gilbert, E. (2017). You can’t stay here: The efficacy of Reddit's 2015 ban examined through hate speech. Proceedings of the ACM on Human-Computer Interaction, 1(CSCW), 1-22. https://doi.org/10.1145/3134666

Chandrasekharan, E., Samory, M., Jhaver, S., Charvat, H., Bruckman, A., Lampe, C., ... Gilbert, E. (2018). The Internet's hidden rules. Proceedings of the ACM on Human-Computer Interaction, 1-25. https://doi.org/10.1145/3274301

Chatzakou, D., Kourtellis, N., Blackburn, J., De Cristofaro, E., Stringhini, G., \& Vakali, A. (2017). Hate is not binary: Studying abusive behavior of \#GamerGate on Twitter. In Proceedings of the 28th ACM Conference on Hypertext and Social Media (pp. 65-74). https://doi.org/10.1145/3078714.3078721

Cohen, K., Johansson, F., Kaati, L., \& Mork, J. C. (2014). Detecting linguistic markers for radical violence in social media. Terrorism and Political Violence, 26(1), 246-256. https://doi.org/10.1080/09546553.2014.849948

Coleman, J., Katz, E., \& Menzel, H. (1966). The diffusion of an innovation among physicians. Sociometry, 20, 253-270. https://doi.org/10.1145/1031607.1031658

Conway, M. (2018). Violent Extremism and Terrorism Online in 2018. Retrieved from https://www.voxpol.eu/year-in-review-2018/

Conway, M., Scrivens, R., \& Macnair, L. (2019). Right-wing extremists' persistent online presence: History and contemporary trends. ICCT Policy Brief. https://doi.org/10.19165/2019.3.12

Crandall, C. S., \& Stangor, C. (2008). Conformity and prejudice. In On the pature of Prejudice; Fifty years after Allport. Blackwell Publishing Ltd.

Davey, J., \& Ebner, J. (2017). The fringe insurgency: Connectivity, convergence and mainstreaming of the extreme right. Retrieved from http://www.isdglobal.org/wp-content/uploads/2017/10/The-FringeInsurgency-221017.pdf

Davidson, T., Warmsley, D., Macy, M., \& Weber, I. (2017). Automated hate speech detection and the problem of offensive language. Proceedings of the Eleventh International AAAI Conference on Web and Social Media (ICWSM 2017), 512-515. Retrieved from http://arxiv.org/abs/1703.04009

de Gibert, O., Perez, N., García-Pablos, A., \& Cuadros, M. (2018). Hate speech dataset from a white supremacy forum. Proceedings Ofthe Second Workshop on Abusive Language Online (ALW2), 11-20. https://doi.org/10.18653/v1/w18-5102

Deveaud, R., SanJuan, E., \& Bellot, P. (2014). Accurate and effective Latent Concept Modeling for ad hoc information retrieval. Document Numerique, 17(1), 61-84. https://doi.org/10.3166/dn.17.1.61-84

Doosje, B., Moghaddam, F. M., Kruglanski, A. W., de Wolf, A., Mann, L., \& Feddes, A. R. (2016). Terrorism, radicalization and de-radicalization. Current Opinion in Psychology, 11, 79-84. https://doi.org/10.1016/j.copsyc.2016.06.008

Dunn, A. G., Surian, D., Dalmazzo, J., Rezazadegan, D., Steffens, M., Dyda, A., ... Mandl, K. D. (2020). Limited role of bots in spreading vaccine-critical information among active Twitter users in the United States: 20172019. AJPH Open-Themed Research, 110(3).

Ekman, M. (2019). Anti-immigration and racist discourse in social media. European Journal of Communication, 34(6), 606-618. https://doi.org/10.1177/0267323119886151 
ElSherief, M., Nilizadeh, S., Nguyen, D., Vigna, G., \& Belding, E. (2018). Peer to peer hate: Hate speech instigators and their targets. ICWSM. Retrieved from http://arxiv.org/abs/1804.04649

Evans, R. (2019). Shitposting, inspirational terrorism, and the Christchurch mosque massacre. Bellingcat. Retrieved from https://www.bellingcat.com/news/rest-of-world/2019/03/15/shitposting-inspirationalterrorism-and-the-christchurch-mosque-massacre/

Fazio, R. H., Eiser, J. R., \& Shook, N. J. (2004). Attitude formation through exploration: Valence asymmetries. Journal of Personality and Social Psychology, 87(3), 293-311. https://doi.org/10.1037/0022-3514.87.3.293

Ferrara, E. (2017). Contagion dynamics of extremist propaganda in social networks. Information Sciences, 418 419, 1-12. https://doi.org/10.1016/j.ins.2017.07.030

Ferrara, E., Wang, W. Q., Varol, O., Flammini, A., \& Galstyan, A. (2016). Predicting online extremism, content adopters, and interaction reciprocity. Lecture Notes in Computer Science (Including Subseries Lecture Notes in Artificial Intelligence and Lecture Notes in Bioinformatics), 10047 LNCS, 22-39. https://doi.org/10.1007/978-3-319-47874-6_3

Finkelstein, J., Zannettou, S., Bradlyn, B., \& Blackburn, J. (2020). A quantitative approach to understanding online Antisemitism. ICWSM. Retrieved from http://arxiv.org/abs/1809.01644

Gagliardone, I., Gal, D., Alves, T., \& Martinez, G. (2015). Countering online hate speech. United Nations Educational, Scientific and Cultural Organization. Retrieved from http://unesdoc.unesco.org/images/0023/002332/233231e.pdf

Gallacher, J. D., \& Heerdink, M. W. (2019). Measuring the effect of Russian Internet research agency information operations in online conversations. Defence Strategic Communications, 6, 155:198. https://doi.org/10.30966/2018.RIGA.6

Gallacher, J. D. (2021). Leveraging cross-platform data to improve automated hate speech detection. ArXiv, 134. Retrieved from https://arxiv.org/abs/2102.04895v1

Gallacher, John D, \& Fredheim, R. E. (2019). Division abroad, cohesion at home: How the Russian troll factory works to divide societies overseas but spread pro-regime messages at home. In Responding to Cognitive Security Challenges (p. 60:79). Riga, Latvia: NATO Strategic Communications Centre of Excellence.

Ganesh, B., \& Bright, J. (2020). Extreme digital speech: Contexts, responses and solutions. Retrieved from https://www.voxpol.eu/new-vox-pol-report-extreme-digital-speech-contexts-responses-and-solutions/

Gaudette, T., Scrivens, R., \& Venkatesh, V. (2020). The role of the Internet in facilitating violent extremism: Insights from former right-wing extremists. Terrorism and Political Violence, 1-18. https://doi.org/10.1080/09546553.2020.1784147

George, D., \& Mallery, P. (2003). SPSS for Windows step by step: A simple guide and reference. Retrieved from https://www.semanticscholar.org/paper/SPSS-for-Windows-Step-by-Step\%3A-A-Simple-Guide-and-GeorgeMallery/230e458b34cdcb463cfe4caa954253bd73456e2e

Glen, S. (2014). Cohen's kappa statistic. Retrieved from https://www.statisticshowto.com/cohens-kappastatistic/

Global Terrorism Index. (2019). Global terrorism index 2019: Measuring the impact of terrorism. Retrieved from https://www.visionofhumanity.org/wp-content/uploads/2020/11/GTI-2019-web.pdf

Graham, E. (2019). Boundary maintenance and the origins of trolling. New Media and Society, 21(9), 2029 -2047. https://doi.org/10.1177/1461444819837561

Granovetter, M. S. (1973). The strength of weak ties. American Journal of Sociology, 78(6), 1360-1380.

Griffiths, T. L., \& Steyvers, M. (2004). Finding scientific topics. Proceedings of the National Academy of Sciences of the United States of America, 101(SUPPL. 1), 5228-5235.

https://doi.org/10.1073/pnas.0307752101

Grover, T., \& Mark, G. (2019). Detecting potential warning behaviors of ideological radicalization in an alt-right subreddit. Proceedings of the 13th International Conference on Web and Social Media, ICWSM 2019, 193204.

Hajjem, M., \& Latiri, C. (2017). Combining IR and and LDA topic modelling for filtering microblogs. Procedia Computer Science, 112, 761-770. https://doi.org/10.1016/j.procs.2017.08.166

Haney, C., Banks, C., \& Zimbardo, P. (1973). Interpersonal dynamics in a simulated prison. International Journal of Criminology and Penology, 1, 69-97. https://doi.org/10.1037/h0076835

Harel, T. O., Jameson, J. K., \& Maoz, I. (2020). The normalization of hatred: Identity, affective polarization, and dehumanization on Facebook in the context of intractable political conflict. Social Media + Society. https://doi.org/10.1177/2056305120913983 
Haslam, S. A., Reicher, S. D., \& Bavel, J. J. Van. (2019). Rethinking the nature of cruelty: The role of identity leadership in the Stanford prison experiment. American Psychologist, 74(7), 809-822.

https://doi.org/10.1037/amp0000443.supp

Hodas, N. O., \& Lerman, K. (2014). The simple rules of social contagion. Scientific Reports, 4. https://doi.org/10.1038/srep04343

Hogg, M. A., Abrams, D., \& Brewer, M. B. (2017). Social identity: The role of self in group processes and intergroup relations. Group Processes and Intergroup Relations, 20(5), 570-581. https://doi.org/10.1177/1368430217690909

Honeycutt, C. (2006). Hazing as a process of boundary maintenance in an online community. Journal of Computer-Mediated Communication, 10(2), 00-00. https://doi.org/10.1111/j.1083-6101.2005.tb00240.x

Ischinger, W. (2020). Munich Security Report 2020. Retrieved from https://securityconference.org/en/

Jagarlamudi, J., Iii, H. D., \& Udupa, R. (2012). Incorporating lexical priors into topic models. EACL 2012 - 13th Conference of the European Chapter of the Association for Computational Linguistics, Proceedings, 204213.

Jasinskaja-Lahti, I., Vezzali, L., Ranta, M., Pacilli, M. G., Giacomantonio, M., \& Pagliaro, S. (2020). Conditional secondary transfer effect: The moderating role of moral credentials and prejudice. Group Processes and Intergroup Relations. https://doi.org/10.1177/1368430220940401

Javed, J., \& Miller, B. (2019). When content promotes hate: Moral-emotional content, outgroup cues, and attitudes toward violence and anti-muslim policies. ArXiv, 1-29. Retrieved from http://www.jeffreyjaved.com/uploads/8/6/1/2/86128854/jm_when_content_promotes_hate_041919.pdf

Johnson, N. F., Leahy, R., Restrepo, N. J., Velasquez, N., Zheng, M., Manrique, P., ... Wuchty, S. (2019). Hidden resilience and adaptive dynamics of the global online hate ecology. Nature, 573(7773), 261-265. https://doi.org/10.1038/s41586-019-1494-7

Katz, R. (2018). Inside the online cesspool of anti-semitism that housed Robert Bowers. Politico. Retrieved from https://www.politico.com/magazine/story/2018/10/29/inside-the-online-cesspool-of-anti-semitism-thathoused-robert-bowers-221949

Kennedy, B., Atari, M., Davani, A. M., Yeh, L., Omrani, A., Kim, Y., ... Dehghani, M. (2020). The Gab hate corpus: A collection of $27 \mathrm{k}$ posts annotated for hate speech. PsyArXiv, 1-47. Retrieved from https://osf.io/edua3/\#!

Kleinberg, B., Vegt, I. Van Der, \& Gill, P. (2020). The temporal evolution of a far-right forum. Journal of Computational Social Science. https://doi.org/10.1007/s42001-020-00064-x

Koehler, D. (2014). The radical online: Individual radicalization processes and the role of the Internet. Journal for Deradicalization, 1(2014), 116-134.

Kramer, A. D. I., Guillory, J. E., \& Hancock, J. T. (2014). Experimental evidence of massive-scale emotional contagion through social networks. Proceedings of the National Academy of Sciences of the United States of America, 111(24), 8788-8790. https://doi.org/10.1073/pnas.1320040111

Kuhn, M., Wickham, H., \& RStudio. (2020). Package 'tidymodels': Easily Install and Load the "Tidymodels" Packages. CRAN, 1-5. Retrieved from https://cran.r-project.org/web/packages/tidymodels/index.html

Lazer, D., Pentland, A., Adamic, L., Aral, S., Barabási, A., Brewer, D., ... Alstyne, M. Van. (2009). Computational Social Science. Science, 323, 721-724.

Lee, B., \& Knott, K. (2020). Fascist aspirants: Fascist forge and ideological learning in the extreme right online milieu. Behavioral Sciences of Terrorism and Political Aggression, 1-25. https://doi.org/10.1080/19434472.2020.1850842

Levine, J. M., \& Moreland, R. L. (1994). Group socialization: Theory and research. European Review of Social Psychology, 5(1), 305-336. https://doi.org/10.1080/14792779543000093

Li, C., Chen, S., Xing, J., Sun, A., \& Ma, Z. (2019). Seed-guided topic model for document filtering and classification. ACM Transactions on Information Systems, 37(1). https://doi.org/10.1145/3238250

Lima, L., Reis, J. C. S., Melo, P., Murai, F., Araujo, L., Vikatos, P., \& Benevenuto, F. (2018). Inside the rightleaning echo chambers: Characterizing Gab, an unmoderated social system. Asonam'18.

MacAvaney, S., Yao, H.-R., Yang, E., Russell, K., Goharian, N., \& Frieder, O. (2019). Hate speech detection: Challenges and solutions. Plos One, 14(8), e0221152. https://doi.org/10.1371/journal.pone.0221152

Makuch, B. (2019). The Nazi-free alternative to Twitter is now home to the biggest far right social network. Vice Motherboard. Retrieved from https://www.vice.com/en/article/mb8y3x/the-nazi-free-alternative-to-twitteris-now-home-to-the-biggest-far-right-social-network 
Mathew, B., Dutt, R., Goyal, P., \& Mukherjee, A. (2019). Spread of hate speech in online social media. Proceedings of the 10th ACM Conference on Web Science - WebSci '19, 173-182. https://doi.org/10.1145/3292522.3326034

McCauley, C., \& Moskalenko, S. (2008). Mechanisms of political radicalization: Pathways toward terrorism. Terrorism and Political Violence, 20(3), 415-433. https://doi.org/10.1080/09546550802073367

McPherson, M., Smith-Lovin, L., \& Cook, J. M. (2001). Birds of a feather: Homophily in social networks. Annual Review of Sociology, 27, 415-444.

McSwiney, J., \& Jasser, G. (2020). Gab.com: The pro-Trump alternive social media. VoxPol. Retrieved from https://www.voxpol.eu/gab-com-the-pro-trump-alternative-to-social-media/

Meleady, R., \& Forder, L. (2019). When contact goes wrong: Negative intergroup contact promotes generalized outgroup avoidance. Group Processes and Intergroup Relations, 22(5), 688-707. https://doi.org/10.1177/1368430218761568

Meleagrou-Hitchens, A., \& Kaderbhai, N. (2016). Research perspectives on online radicalization. VOX-Pol Network of Excellence. Retrieved from https://icsr.info/wp-content/uploads/2017/05/ICSRPaper_Research-Perspectives-on-Online-Radicalisation-A-Literature-Review-2006-2016.pdf

Milgram, S. (1963). Behavioral study of obedience. Journal of Abnormal Psychology, 67(4), 371-378. https://doi.org/10.1037/h0040525

Mønsted, B., Sapieżyński, P., Ferrara, E., \& Lehmann, S. (2017). Evidence of complex contagion of information in social media: An experiment using Twitter bots. PLoS ONE, 12(9), 1-12. https://doi.org/10.1371/journal.pone.0184148

Moreland, R. L., \& Levine, J. M. (1982). Socialization in small groups: Temporal changes in individual-group relations. Advances in Experimental Social Psychology, 15(C), 137-192. https://doi.org/10.1016/S00652601(08)60297-X

Nielsen, J. (2006). The 90-9-1 rule for participation inequality in social media and online communities. Nielsen Norman Group, 5-9. Retrieved from https://www.nngroup.com/articles/participation-inequality/

Nikita, M. (2016). Select number of topics for LDA model. RPubs. Retrieved from https://rpubs.com/siri/ldatuning

Noel, J. G., Wann, D. L., \& Branscombe, N. R. (1995). Peripheral ingroup membership status and public negativity toward outgroups. Journal of Personality and Social Psychology, 68(1), 127-137. https://doi.org/10.1037/0022-3514.68.1.127

Nonnecke, B. (2000). Lurking in email-based discussion lists. South Bank University.

O'Callaghan, D., Greene, D., Conway, M., Carthy, J., \& Cunningham, P. (2015). Down the (white) rabbit hole: The extreme right and online recommender systems. Social Science Computer Review, 33(4), 459-478. https://doi.org/10.1177/0894439314555329

Olteanu, A., Castillo, C., Boy, J., \& Varshney, K. R. (2018). The effect of extremist violence on hateful speech online. ArXiv. Retrieved from http://arxiv.org/abs/1804.05704

Onnela, J. P., \& Reed-Tsochas, F. (2010). Spontaneous emergence of social influence in online systems. Proceedings of the National Academy of Sciences of the United States of America, 107(43), 18375-18380. https://doi.org/10.1073/pnas.0914572107

Pariser, E. (2011). The Filtter Bubble: What the internet is hiding from you. New York, New York, USA: The Penguin Press.

Park, J. H., \& Fung, P. (2017). One-step and two-step classification for abusive language detection on Twitter. ArXiv. https://doi.org/10.18653/v1/w17-3006

Pauwels, L., \& Schils, N. (2016). Differential online exposure to extremist content and political Violence: Testing the relative strength of social learning and competing perspectives. Terrorism and Political Violence, 28(1), 1-29. https://doi.org/10.1080/09546553.2013.876414

Pettigrew, T. F. (1997). Generalized intergroup contact effects on prejudice. Personality and Social Psychology Bulletin, 23(2), 173-185. https://doi.org/10.1177/0146167297232006

Pettigrew, T. F. (2009). Secondary transfer effect of contact: Do intergroup contact effects spread to noncontacted outgroups? Social Psychology, 40(2), 55-65. https://doi.org/10.1027/1864-9335.40.2.55

Pettigrew, T. F., \& Tropp, L. R. (2008). How does intergroup contact reduce prejudice? Meta-analytic tests of three mediators. European Journal of Social Psychology, 38, 922-934. https://doi.org/10.1002/ejsp

Preoţiuc-Pietro, D., Liu, Y., Hopkins, D., \& Ungar, L. (2017). Beyond binary labels: Political ideology prediction of Twitter users. Proceedings Ofthe 55th Annual Meeting Ofthe Association for Computational Linguistics, 
729-740. https://doi.org/10.18653/v1/p17-1068

Rajadesingan, A., Resnick, P., \& Budak, C. (2020). Quick, community-specific learning: How distinctive toxicity norms are maintained in political subreddits. Proceedings of the International AAAI Conference on Web and Social Media, 14. Retrieved from https://www.aaai.org/ojs/index.php/ICWSM/article/view/7323

Reed, A., Whittaker, J., Votta, F., \& Looney, S. (2019). Radical filter bubbles and extremist content. Global Research Network on Terrorism and Technology, (8). Retrieved from https://rusi.org/sites/default/files/20190726_grntt_paper_08_0.pdf

Reicher, S., Haslam, S. A., \& Rath, R. (2008). Making a virtue of evil: A five-step social identity model of the development of collective hate. Social and Personality Psychology Compass, 2(3), 1313-1344. https://doi.org/10.1111/j.1751-9004.2008.00113.x

Ribeiro, M. H., Blackburn, J., Bradlyn, B., De Cristofaro, E., Stringhini, G., Long, S., ... Zannettou, S. (2020). The evolution of the manosphere across the web. ArXiv. Retrieved from http://arxiv.org/abs/2001.07600

Ribeiro, M. H., Calais, P. H., Santos, Y. A., Almeida, V. A. F., \& Meira, W. (2018). Characterizing and detecting hateful users on Twitter. 12th International AAAI Conference on Web and Social Media, ICWSM 2018, 676-679. Retrieved from https://arxiv.org/pdf/1803.08977.pdf

Robbinson, N., Schlegel, L., Janin, M., \& Deverell, F. (2020). How are terrorists and violent extremists using gamification? Retrieved July 17, 2020, from https://www.techagainstterrorism.fm/how-are-terrorists-andviolent-extremists-using-gamification/

Rogers, E. M. (2003). Diffusion of Innovations. The Free Press.

Rogers, E. M., \& Kincaid, L. (1984). Communication networks: Toward a new paradigm for research. American Journal of Sociology, 89(4), 986-988. https://doi.org/10.1086/227967

Rowe, M., \& Saif, H. (2016). Mining pro-ISIS radicalisation signals from social media users. Proceedings of the 10th International Conference on Web and Social Media, ICWSM 2016, 329-338. Retrieved from http://oro.open.ac.uk/48477/1/13023-57822-1-PB.pdf

Ryan, B., \& Gross, N. C. (1943). Acceptance and diffusion of hybrid corn seed in two Iowa communities. Agricultural Experiment Station - Iowa State College of Agriculture and Mechanic Arts, 372(372), 663-705. Retrieved from

http://ezproxy.cul.columbia.edu/login?url=http://search.ebscohost.com/login.aspx?direct=true\&db=ehh\& $\mathrm{AN}=13074695 \&$ site $=$ ehost-live\&scope $=$ site

Sageman, M. (2004). Understanding terror networks. University of Pennsylvania Press.

Sarrasin, O., Fasel, N., Green, E. G. T., \& Helbling, M. (2015). When sexual threat cues shape attitudes toward immigrants: the role of insecurity and benevolent sexism. Frontiers in Psychology, 6(July), 1-13. https://doi.org/10.3389/fpsyg.2015.01033

Schmid, K., Hewstone, M., \& Tausch, N. (2014). Secondary transfer effects of intergroup contact via social identity complexity. British Journal of Social Psychology, 53(3), 443-462. https://doi.org/10.1111/bjso.12045

Schofield, A., Magnusson, M., Thompson, L., \& Mimno, D. (2017). Understanding text pre-processing for Latent Dirichlet Allocation. Proceedings of the 15th Conference of the European Chapter of the Association for Computational Linguistics, 2, 432-436. https://doi.org/10.1145/1378773.1378800

Scrivens, R. (2020). Exploring radical right-wing posting behaviors online. Deviant Behavior, 1-15. https://doi.org/10.1080/01639625.2020.1756391

Scrivens, R., Wojciechowski, T. W., \& Frank, R. (2020). Examining the developmental pathways of online posting behavior in violent right-wing extremist forums. Terrorism and Political Violence, 1-18. https://doi.org/10.1080/09546553.2020.1833862

Settle, J. E. (2018). Frenemies. Cambridge University Press. https://doi.org/10.1017/9781108560573

Shalizi, C. R., \& Thomas, A. C. (2011). Homophily and contagion are generically confounded in observational social network studies. Sociological Methods and Research, 40(2), 211-239. https://doi.org/10.1177/0049124111404820

Sherif, M. (1936). The psychology of social norms. Harper.

Silge, J., \& Robinson, D. (2017). Text mining with R: a tidy approach. Sebastopol: O'Reilly Media.

Singhal, A. (2001). Modern information retrieval: A brief overview. Bulletin of the IEEE Computer Society Technical Committee on Data Engineering. https://doi.org/10.1350/ijps.2005.7.3.197

Smith, L. G. E., Blackwood, L., \& Thomas, E. F. (2019). The need to refocus on the group as the site of radicalization. Perspectives on Psychological Science, 15(2), 327-352. 
https://doi.org/10.1177/1745691619885870

Soral, W., Bilewicz, M., \& Winiewski, M. (2018). Exposure to hate speech increases prejudice through desensitization. Aggressive Behavior, 44(2), 136-146. https://doi.org/10.1002/ab.21737

Susarla, A., Oh, J. H., \& Tan, Y. (2012). Social networks and the diffusion of user-generated content: Evidence from youtube. Information Systems Research, 23(1), 23-41. https://doi.org/10.1287/isre.1100.0339

Tajfel, H. (1974). Social identity and intergroup behaviour. Social Science Information, 13(2), 65-93. https://doi.org/10.1177/053901847401300204

Tausch, N., Hewstone, M., Kenworthy, J. B., Psaltis, C., Schmid, K., Popan, J. R., ... Hughes, J. (2010). Secondary transfer effects of intergroup contact: Alternative accounts and underlying processes. Journal of Personality and Social Psychology, 99(2), 282-302. https://doi.org/10.1037/a0018553

Terizi, C., Chatzakou, D., Pitoura, E., Tsaparas, P., \& Kourtellis, N. (2020). Angry birds flock together: Aggression propagation on social media. ArXiv. Retrieved from http://arxiv.org/abs/2002.10131

Theocharis, Y., Barberá, P., Fazekas, Z., \& Popa, S. (2019). The dynamics of political incivility on Twitter. SAGE Open, 1-15. https://doi.org/10.1177/ToBeAssigned

Thomas, E. F., Mcgarty, C., \& Louis, W. (2014). Social interaction and psychological pathways to political engagement and extremism. European Journal of Social Psychology, 44(1), 15-22. https://doi.org/10.1002/ejsp.1988

Tuters, M., \& Hagen, S. (2018). Who are (((they)))?: On online hate, tasteless transgression, and memetic versatility. Retrieved from https://oilab.eu/who-are-they-on-online-hate-tasteless-transgression-andmemetic-versatility/

Tuters, M., \& Hagen, S. (2019). (((They))) rule: Memetic antagonism and nebulous othering on 4chan. New Media and Society, 1-20. https://doi.org/10.1177/1461444819888746

Urman, A., \& Katz, S. (2020). What they do in the shadows: examining the far-right networks on Telegram. Information Communication and Society, O(0), 1-20. https://doi.org/10.1080/1369118X.2020.1803946

Valente, T. W. (1995). Network models of the diffusion of innovations. Hampton Press.

Valente, T. W. (1996). Network models of the diffusion of innovations. Computational and Mathematical Organization Theory, 2.

Valente, T. W., Dyal, S. R., Chu, K. H., Wipfli, H., \& Fujimoto, K. (2015). Diffusion of innovations theory applied to global tobacco control treaty ratification. Social Science and Medicine, 145, 89-97. https://doi.org/10.1016/j.socscimed.2015.10.001

Valente, T. W., \& Vega Yon, G. G. (2020). Diffusion/contagion processes on social networks. Health Education and Behavior, 47(2), 235-248. https://doi.org/10.1177/1090198120901497

Van Kleef, G. A. (2009). How emotions regulate social life. Current Directions in Psychological Science, 18(3), 184-188. https://doi.org/10.1111/j.1467-8721.2009.01633.x

Van Kleef, G. A., Van Den Berg, H., \& Heerdink, M. W. (2015). The persuasive power of emotions: Effects of emotional expressions on attitude formation and change. Journal of Applied Psychology, 100(4), 1124-1142. https://doi.org/10.1037/apl0000003

Vega Yon, G., Valente, T., Dyal, S., \& Hayes, T. (2020). Package 'netdiffuseR': Analysis of diffusion and contagion processes on networks. CRAN. https://doi.org/10.1086/303110

Vidgen, B., Yasseri, T., \& Margetts, H. (2019). Trajectories of Islamophobic hate amongst far right actors on Twitter. ArXiv, 1-20. Retrieved from https://arxiv.org/pdf/1910.05794

Weich, B. (2019). Inside Gab, the alt-right's social media network that is awash with antisemitism. The Jewish Chronicle. Retrieved from https://www.thejc.com/news/world/inside-the-social-media-network-of-the-altright-1.477090

Weissman, C. G. (2016). Inside Gab: The new Twitter alternative championed by the alt-right. Fast Company. Retrieved from https://www.fastcompany.com/3065777/inside-gab-the-new-twitter-alternative-championedby-the-alt-right

Wickham, H., Averick, M., Bryan, J., Chang, W., D', L., Mcgowan, A., ... Yutani, H. (2019). Welcome to the Tidyverse. Journal of Open Source Software, 4(43), 1686. https://doi.org/10.21105/joss.01686

Williams, M. L., \& Burnap, P. (2016). Cyberhate on Social Media in the aftermath of Woolwich: A Case Study in Computational Criminology and Big Data. British Journal of Criminology, 56(2), 211-238. https://doi.org/10.1093/bjc/azv059

Williams, M. L., Burnap, P., Javed, A., Liu, H., \& Ozalp, S. (2019). Hate in the machine: Anti-black and antimuslim social media posts as predictors of offline racially and religiously aggravated crime. The British 
Journal of Criminology, 60(1), 1-25. https://doi.org/10.1093/bjc/azz049

Williams, M., \& Mishcon de Reya. (2019). Hatred behind the screens A report on the rise of online hate speech. Retrieved from https://www.mishcon.com/upload/files/Online Hate Final 25.11.pdf

Wood, S. (2019). Package "mgcv": Mixed GAM computation vehicle with automatic smoothness estimation. CRAN. https://doi.org/10.1201/9781315370279>

Zannettou, S., Bradlyn, B., De Cristofaro, E., Kwak, H., Sirivianos, M., Stringhini, G., \& Blackburn, J. (2018). What is Gab? A bastion of free speech or an alt-right echo chamber? ArXiv. https://doi.org/10.1145/3184558.3191531

Zannettou, S., ElSherief, M., Belding, E., Nilizadeh, S., \& Stringhini, G. (2020). Measuring and characterizing hate speech on news websites. ArXiv. Retrieved from http://arxiv.org/abs/2005.07926

Zhou, Y., Dredze, M., Broniatowski, D. A., \& Adler, W. D. (2019). Elites and foreign actors among the alt-right: The Gab social media platform. First Monday. https://doi.org/10.5210/fm.v24i9.10062

Zuo, Y., Wu, J., Zhang, H., Lin, H., Wang, F., Xu, K., \& Xiong, H. (2016). Topic modeling of short texts: A pseudo-document view. In Proceedings of the ACM SIGKDD International Conference on Knowledge Discovery and Data Mining (pp. 2105-2114). https://doi.org/10.1145/2939672.2939880 
Supplementary Information (SI) for:

Hate Contagion: Measuring the spread and trajectory of hate on social media

1 - Hate speech classification

1.1 Hate classification processing pipeline

1.2 Hate speech topic modelling / target of hate detection

2 - Conversation dynamics on Gab

2.1 Daily hate density over time

2.2 Daily conversation rhythm

3 - Additional hate speech trajectory analysis

3.1 Robustness checks for longer term trends

3.2 Removing low activity users

4 - Distribution of hate across users

5 - Short-term exposure effects of hate speech exposure when controlling for longer term exposure trends

6 - Daily example of short-term social contagion 


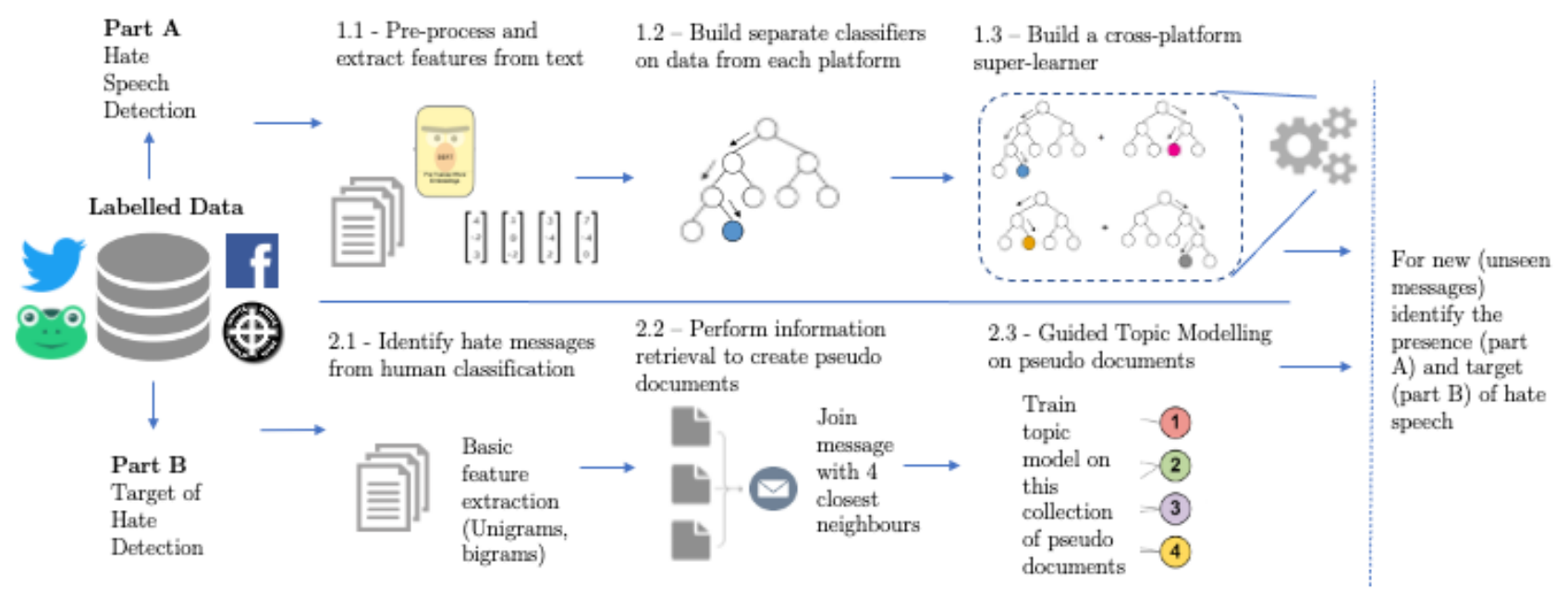

SI Figure 1 - Modelling pipeline for hate speech detection (A) and type of hate classification (B)

\section{1 - Hate speech classification}

\section{1 - Hate classification processing pipeline}

SI Figure 1 shows the processing pipeline for hate speech detection. Part A gives an overview of how hate speech is detected in social media messages. See Gallacher, 2021 for more details on this step.

\section{2 - Hate speech topic modelling / target of hate detection}

In order to identify the group which is targeted within a hate speech message we use a form of semisupervised machine learning called guided topic modelling (Anoop et al., 2016; Li et al., 2019). The steps in this process are shown in SI Figure 1 part B, and detailed below.

\section{Data pre-processing}

Prior to training any topic models we filter out stop words from the messages (linking words without any semantic value, e.g., "and"), make all words lowercase, but avoid stemming the words as is traditional, as this has been found more recently to be detrimental to topic modelling using LDA (Schofield, Magnusson, Thompson, \& Mimno, 2017). We then extract both unigrams (single words) and bigrams (word pairs) from the messages and cast the dataset to a document term matrix (SI Figure 12.1$)$.

Topic modelling on short texts such as social media messages is notoriously difficult because of the low co-occurrence of words within the messages (Zuo et al., 2016). To overcome this, we use an information retrieval approach with a pseudo-message model. When training the model, for each message across the corpus, we first gather the top four most similar messages to it from across the corpus (see below), and join these together to form a longer 'pseudo-message'. This approach increases the word co-occurrence within each topic and builds greater stability of the topics (Hajjem \& Latiri, 2017; Zuo et al., 2016), and performs well on short messages such as those on Twitter or Gab (Gallacher \& Fredheim, 2019). The similarity of messages is identified by creating a matrix of termfrequency/inverse document frequency (tf-idf) values for all unigrams \& bigrams in the messages, and 


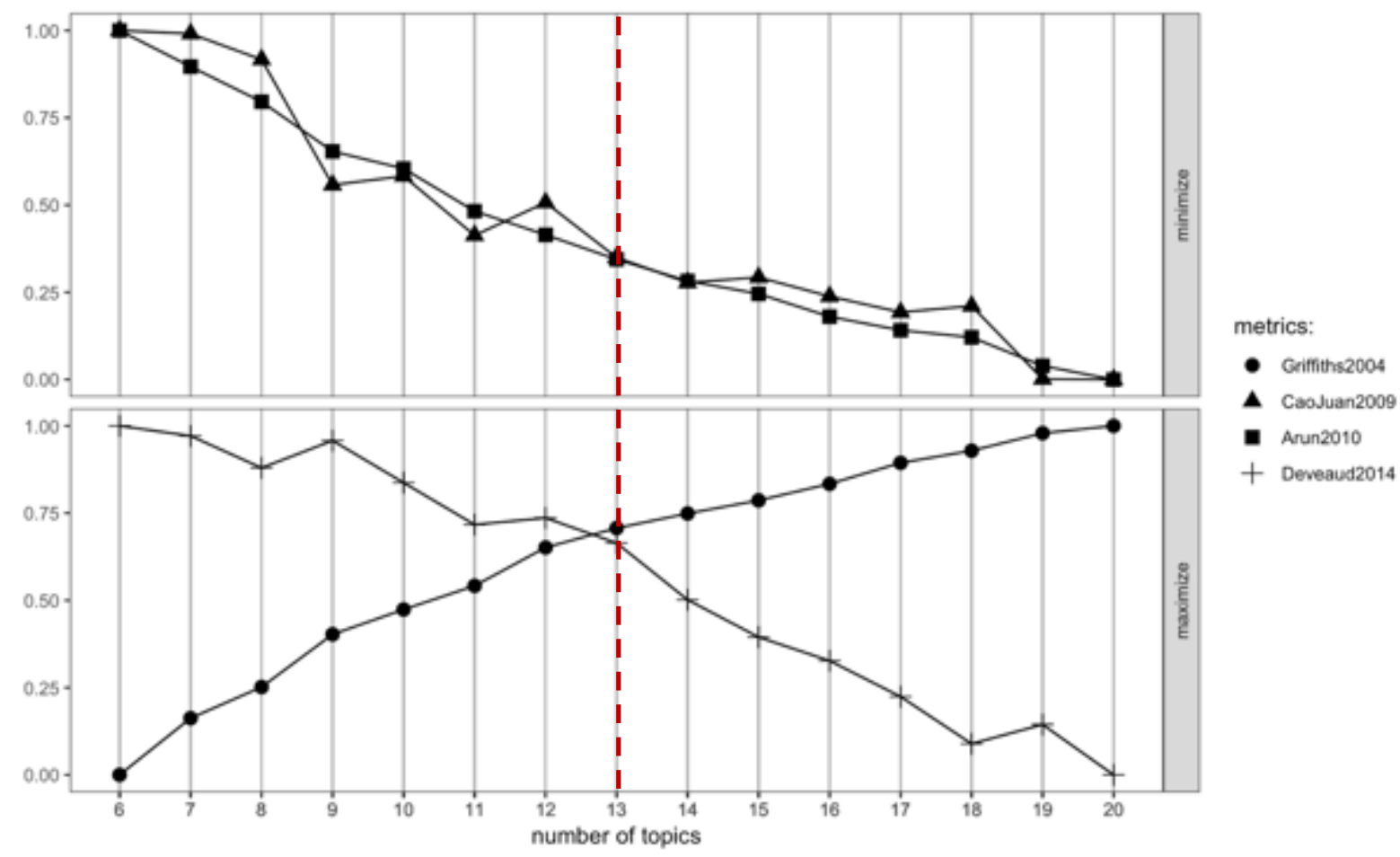

SI Figure 2 - LDA tuning to select the appropriate number of topics

calculating the cosine similarity of messages (Singhal, 2001). These pseudo-messages are then treated as the documents for topic modelling (SI Figure 1, 2.2).

\section{Selecting the optimum number of topics}

Latent Dirichlet Allocation (LDA) approaches for topic modelling demand that the number of topics (here, the number of types of hate speech) is identified ahead of the modelling process. This creates a challenge when we do not know how many topics exist within the dataset. In order to identify this in a more structured way, we use the 'ldatuning' approach (Nikita, 2016), whereby multiple LDA models are created with topics numbering from 2-20 and four different measures of model performance are computed (Arun, Suresh, Madhavan, \& Murthy, 2010; Cao, Xia, Li, Zhang, \& Tang, 2009; Deveaud, SanJuan, \& Bellot, 2014; Griffiths \& Steyvers, 2004). The optimum number of topics is then defined as the value which maximally satisfies all 4 of the measures. For these 19 models we select alpha levels (higher alpha indicates that messages are assumed to be made up of greater mixture of topics) equal to 20 / the number of topics (k) as per the literature (Blei et al., 2003). This method identifies an optimal number of 13 topics as shown in SI Figure 2.

We then manually classify these topics into the type of hate that they represent using the top words (Figure 1) and the most confident judgements (messages with the highest gamma score) per topic. Where multiple of these topics covered the same type of hate, often with subtly different angles, they were combined, leaving 11 distinct topics (Table 2). 


\section{2 - Conversation dynamics on Gab}

\section{1 - Daily hate density over time}

SI Figure SI 3 shows the overall proportion of messages which were classified as hate speech (daily hate density) across Gab over the entire collection periods. The results show that there was considerable variation in the amount of hate posted on different days, with some days having much higher densities of hate in the platform conversations than others.

On Gab we find that the density of hate speech quickly rises from the formation of the platform August 2016 before reaching a plateau in January 2017 and then climbing only slightly from here onwards. These trends highlight the importance of accounting for the overall level of hate speech on the platform when looking at individual user trajectories.

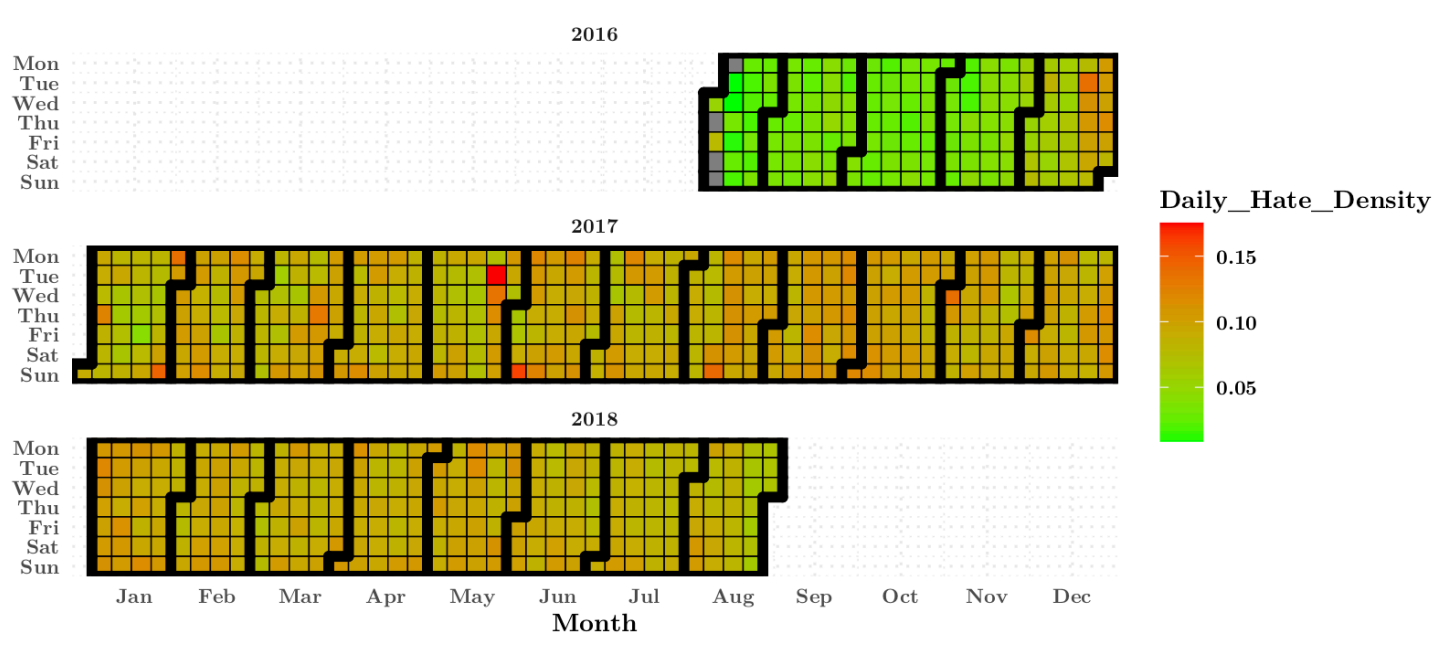

Si Figure 3 - Daily proportions of hate speech across (A) Facebook and (B) Gab 


\section{2 - Daily conversation rhythm}

Daily Gab conversations followed a consistent pattern with a peak of activity between $1600 \mathrm{hrs}$ and $2000 \mathrm{hrs}$ and a minimum at $0700 \mathrm{hrs}$ (SI Figure 4). Because of this we created breaks in the network at $0700 \mathrm{hrs}$, treating each intervening period as a single 'daily conversation'. This was chosen as breaking the networks at $0000 \mathrm{hrs}$ (midnight) would have been near the peak activity, so using 0700hrs instead would lead to minimal conversation broken across days.

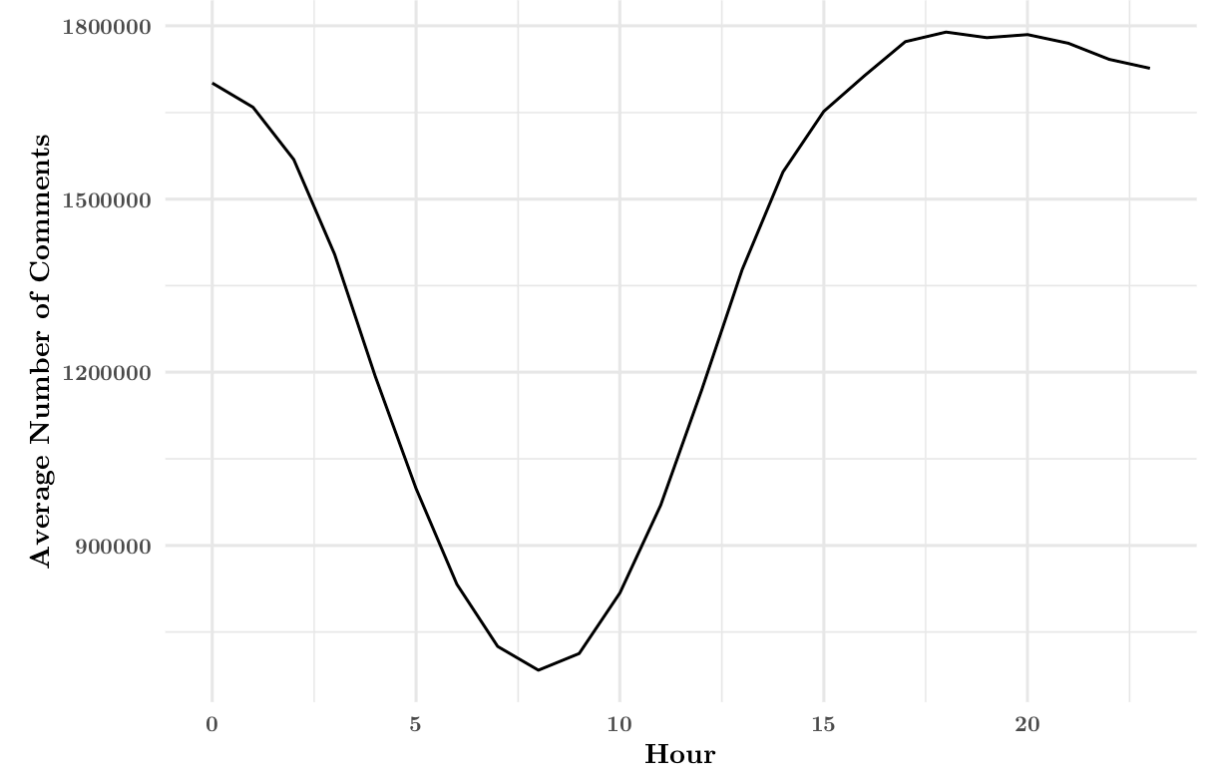

SI Figure 4 - Daily Gab conversation rhythm with a clear minimum at 0700hrs

\section{3 - Additional hate speech trajectory analysis}

\section{1 - Robustness checks for longer term trajectory trends on Gab \& log} adjusting the user post index for the trajectory models

SI Figure 5a shows the average hate trajectory for users on Gab over the period of their first to their $2150^{\text {th }}$ post when controlling for overall density of hate speech on the platform and inter-user differences. Hate increased over the first $\sim 1,000$ posts before plateauing (GAMM, n = 18,159,111, df $=$ $1.533, \mathrm{~F}=153.3, \mathrm{p}<0.001)$

SI Figure 5b shows the log-adjusted trajectory of likelihood of hate expression for users on Gab. Taking the log of the user post index reduces the impact of users dropping out from the conversations at the later stages. We find that the overall shape of the trajectory doesn't change by taking the log of the user post index and is broadly comparable- an initial higher chance of a user posting hate when they first join the platform, followed by a decrease before this reverses and likelihood of expressing hate rises over the longer term (GAMM, $\mathrm{n}=18,159,111, \mathrm{df}=1.563, \mathrm{~F}=169.1, \mathrm{p}<0.001$ ) 


\section{2 - Removing low activity users}

SI Figure SI 6 shows the user hate trajectories after removing users who made fewer than 250 posts to the platform, and retaining over the 'very active users'. We model this over 250 and 2150 posts. In both cases a significant relationship between the user post index and hate speech probability was found (Gab 2150; $\mathrm{n}=15,209,090, \mathrm{df}=1.61, \mathrm{~F}=11.64, \mathrm{p}<0.001$, Gab 250; $\mathrm{n}=3,764,000, \mathrm{df}=$ $2.571, \mathrm{~F}=16.8, \mathrm{p}<0.001)$

(A) Hate trajectories for messages 1-2150

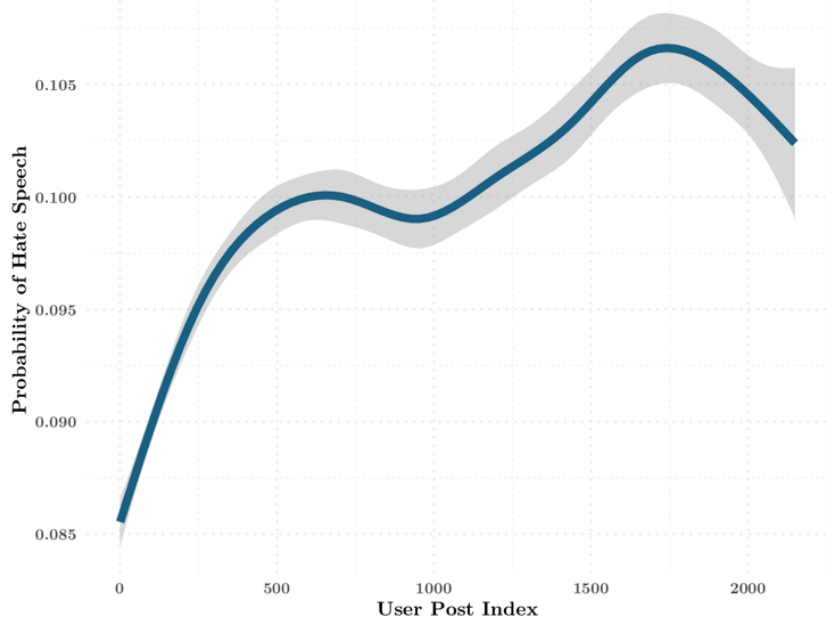

(B) Log adjusted hate trajectories 1-2150

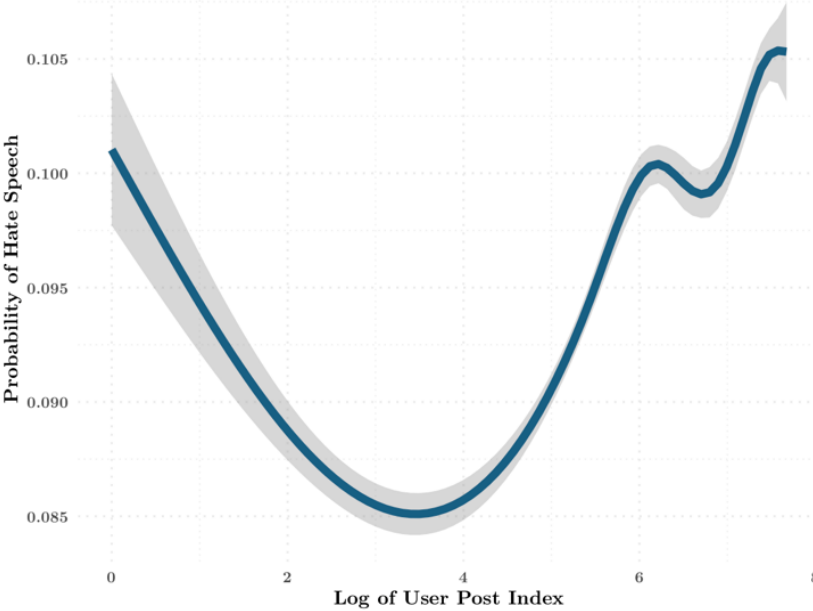

SI Figure 5 - (A) Long-term hate trajectory for users on Gab for 1 - 2150 posts, (B) Log-adjusted user hate trajectories over this same period

(A) Post Index 1-250: Very Active Users

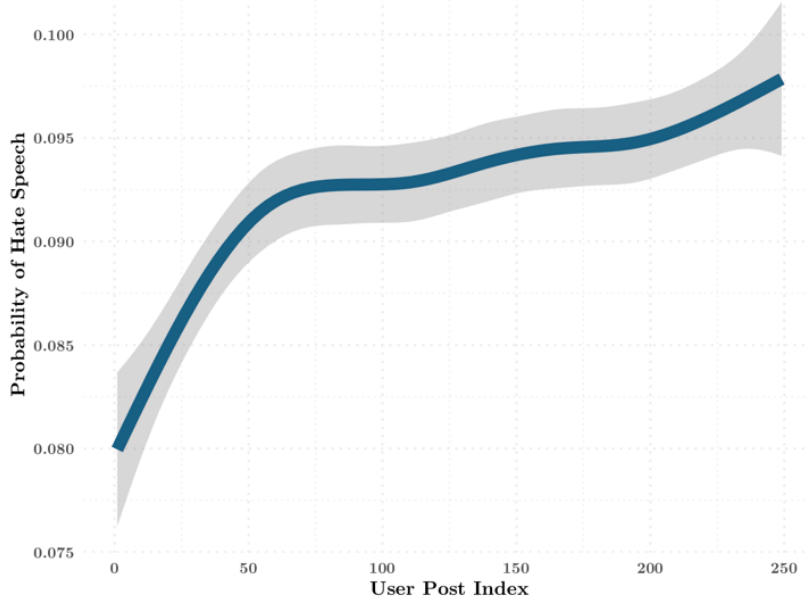

(B) Post Index 1-2150: Very Active Users

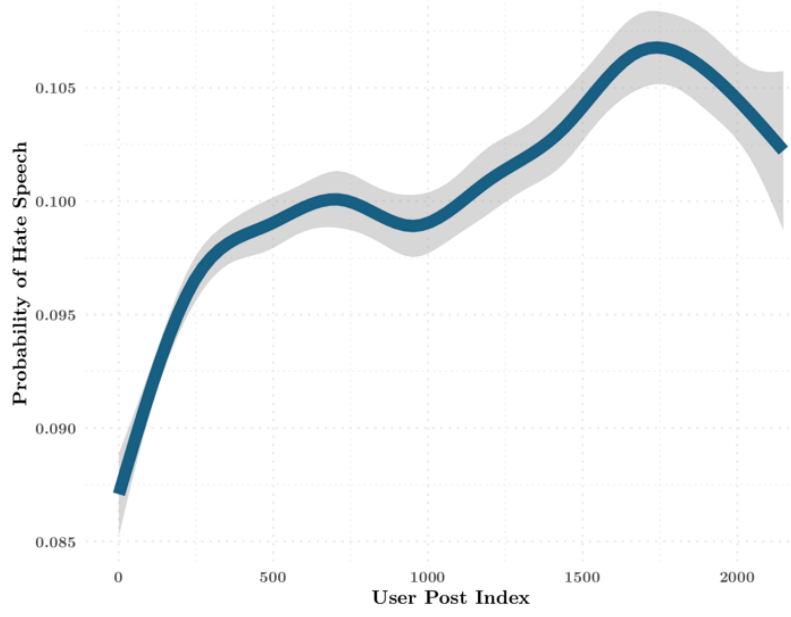

SI Figure 6 - User hate trajectories for 'very active users' who post over 250 times over (A) their first 250 posts and (B) their first 2150 posts 


\section{4 - Distribution of hate across users}

SI Figure 7 shows the Lorentz curve and the Gini coefficient for hate speech production on Gab. The Gini Coefficient estimates the degree to which hate speech messages are concentrated on a small subset of users as a value between 0 (perfect equality between users) and 1 (one user posting all the hate). This method revealed the dominance of a few 'superusers' in far-right forums (Kleinberg et al., 2020). If all users contributed equally to hate speech on a platform, the cumulative percentage of hate speech posts would increase linearly with the cumulative percentage of users. Conversely, if hate is expressed unevenly, a small proportion of users would produce a large proportion of the hate speech.

We find that hate was very unevenly distributed across users, with a few 'hateful superusers' posting the majority of hate (Gini coefficient $=0.97$ ). This means that $50.0 \%$ of hate speech posts were generated by only $0.05 \%$ of users

\section{Lorenz curve for Gab}

Gini coefficient is 0.97

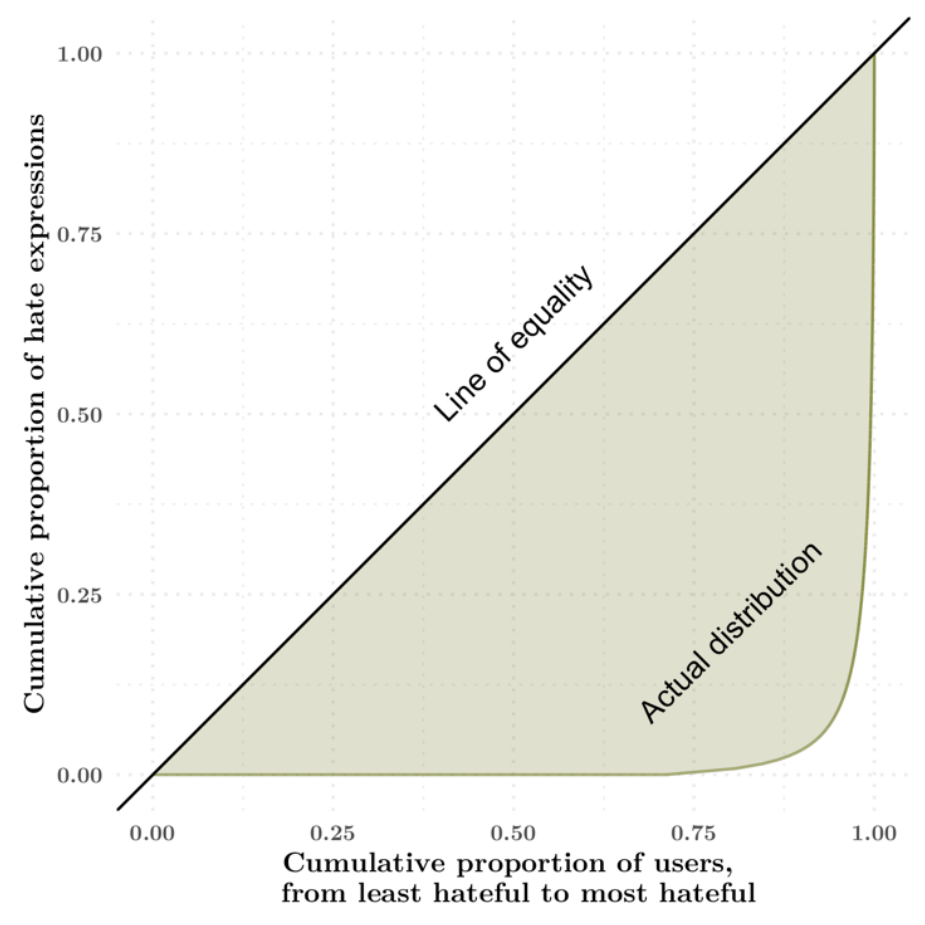

SI Figure 7 - Lorenz curves showing inequality among users for hate speech production for Gab 


\section{5 - Short-term exposure effects of hate speech exposure when controlling for longer term exposure trends}

SI Table 1 gives the short-term effects of hate speech exposure once controlling for longer-term effects of hate speech exposure over the prior seven days.

SI Table 1 - Short-term (daily, pre-time of adoption) effects of exposure to hate speech once accounting for longer 7day effects

\begin{tabular}{|c|c|c|c|c|c|c|c|c|c|c|c|c|c|c|c|c|c|c|}
\hline \multirow{2}{*}{$\begin{array}{l}\text { BEHAVIOUR } \\
\text { (HATE TYPE) }\end{array}$} & \multicolumn{3}{|c|}{ Anti-Semitism } & \multicolumn{3}{|c|}{ Islamophobia } & \multicolumn{3}{|c|}{ Misogyny } & \multicolumn{3}{|c|}{ Anti Black Racism } & \multicolumn{3}{|c|}{ Anti Immigration } & \multicolumn{3}{|c|}{ Homophobia } \\
\hline & ESTIMATE & F & $P$ & ESTIMATE & $\mathrm{F}$ & $P$ & ESTIMATE & F & $P$ & ESTIMATE & F & $P$ & ESTIMATE & F & $P$ & ESTIMATE & F & $P$ \\
\hline \multicolumn{19}{|c|}{ Daily exposure pre-toa } \\
\hline Anti-Semitism & 8.52 & 3.19 & 0.01 & 3.60 & 2.50 & 0.10 & 6.57 & 0.68 & 1.00 & 4.34 & 0.14 & 1.00 & 2.78 & 1.19 & 1.00 & 5.02 & 0.12 & 1.00 \\
\hline Islamophobia & 1.34 & 0.92 & 0.40 & 2.09 & 2.13 & 0.16 & -9.85 & -3.54 & 0.00 & 6.34 & 2.94 & 0.02 & -2.64 & -1.51 & 0.39 & -9.75 & -2.90 & 0.02 \\
\hline Misogyny & 9.78 & 2.16 & 0.33 & 9.53 & 2.84 & 0.05 & -3.06 & -0.20 & 1.00 & 20.02 & 1.64 & 0.83 & -20.48 & -2.17 & 0.33 & -46.39 & -1.56 & 0.84 \\
\hline $\begin{array}{l}\text { Anti-Black } \\
\text { Racism }\end{array}$ & -6.53 & -1.17 & 1.00 & 10.54 & 3.64 & 0.00 & -3.23 & -0.37 & 1.00 & -21.33 & -1.98 & 0.48 & -12.13 & -1.59 & 0.89 & 7.34 & 0.66 & 1.00 \\
\hline $\begin{array}{c}\text { Anti- } \\
\text { Immigration }\end{array}$ & 2.14 & 0.92 & 1.00 & 5.93 & 4.49 & 0.00 & 6.05 & 0.89 & 1.00 & 2.21 & 0.24 & 1.00 & 6.32 & 2.67 & 0.07 & -5.66 & -0.80 & 1.00 \\
\hline Homophobia & -15.23 & -1.94 & 0.41 & 11.40 & 3.37 & 0.01 & 0.65 & 0.05 & 1.00 & 21.99 & 2.42 & 0.15 & -6.96 & -0.99 & 1.00 & 26.72 & 3.43 & 0.01 \\
\hline
\end{tabular}

\section{6 - Daily example of short-term social contagion}

SI Figure 8 shows network slices for a randomly selected day in the dataset (31 ${ }^{\text {st }}$ March 2018). Users (nodes) and edges (connections) are coloured by their adoption status. From this we estimate the role of connections in shaping the diffusion of behaviours (hate speech expression) through the network.

SI Figure 9 gives the diffusion curve for this contagion pattern. The S-shaped results indicate that early in the diffusion process few people adopt the behaviour, but over time more individuals adopt the behaviour until this reaches a natural plateau / saturation point which is $\sim 30 \%$ in this example.

Running a structural dependency test for this day indicates that the network influences the diffusion of behaviour at a level much higher than chance. This tests whether or not a network estimates can be considered a function of the network structure i.e. whether connections and patterns of exposure explain the observed behaviours. By rewiring the graph and calculating a particular statistic t, the test compares the observed mean of $t$ against the empirical distribution of it obtained from rewiring the network using Monte Carlo simulations. (Simulations $=1,000$, nodes $=861$, time periods $=24$, observed $=0.2839$, expected $=0.0004, \mathrm{p}<0.001)$

SI Table 2 gives the spatial autocorrelation values (Moran's I) for each slice in the network along with the adoption rate. 

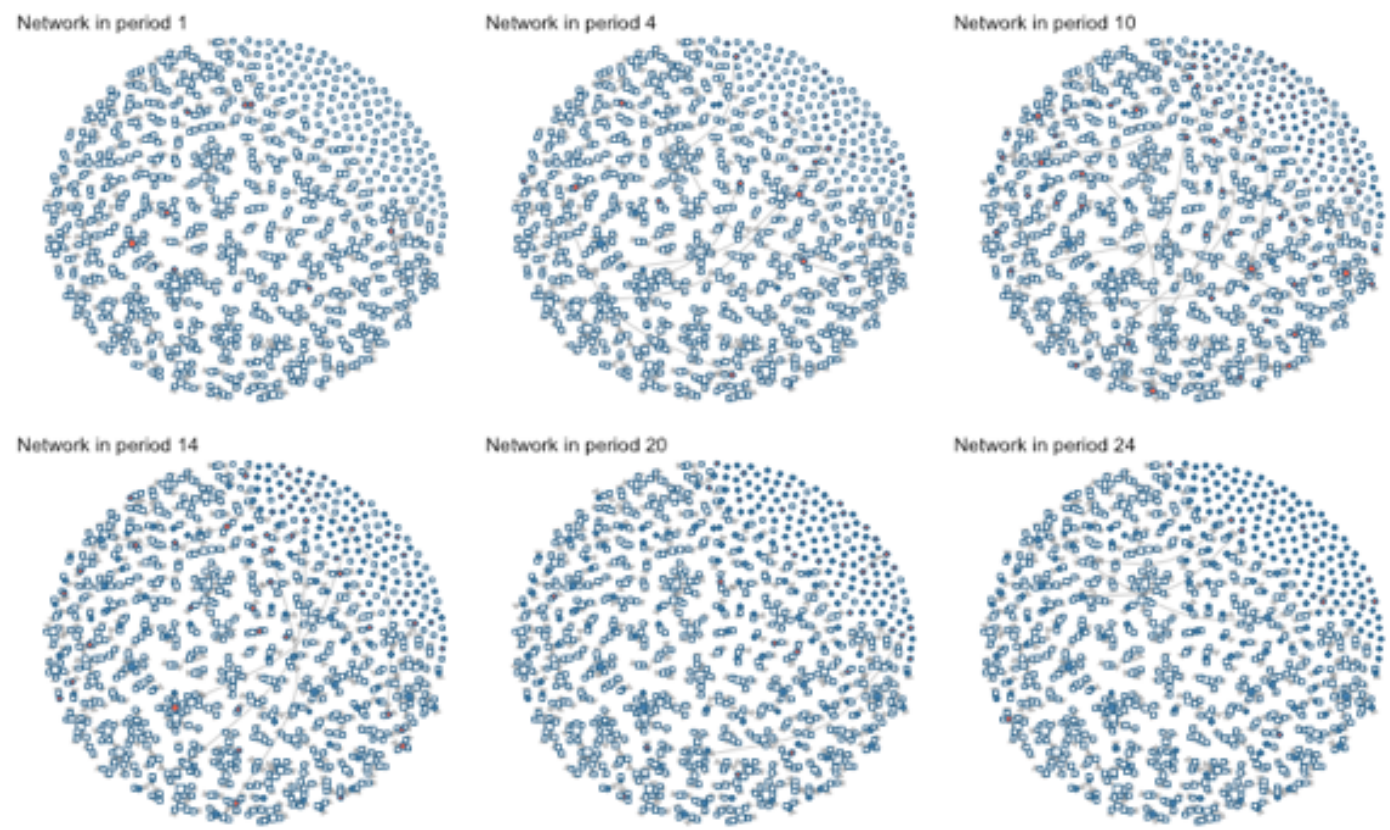

Network in period 20

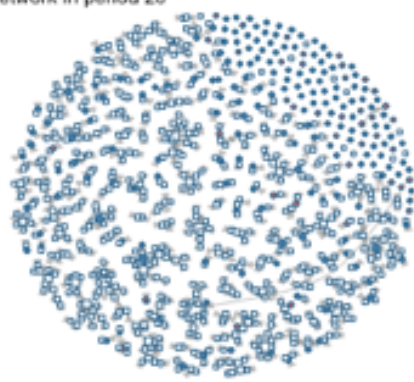

Network in period 24

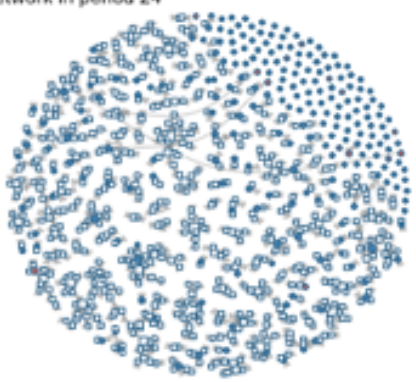

- Non adopters

- New adopters

Adopters

SI Figure 8 - Example diffusion effects for a single day in the dataset (31 ${ }^{\text {st }}$ March 2018) across six out of the 24 network slices. Users are shown as nodes, with edges representing network connections in the form of replies, @mentions and re-posts. Nodes are coloured according to their hate adoption state.

\section{Adopters and Cumulative Adopters}

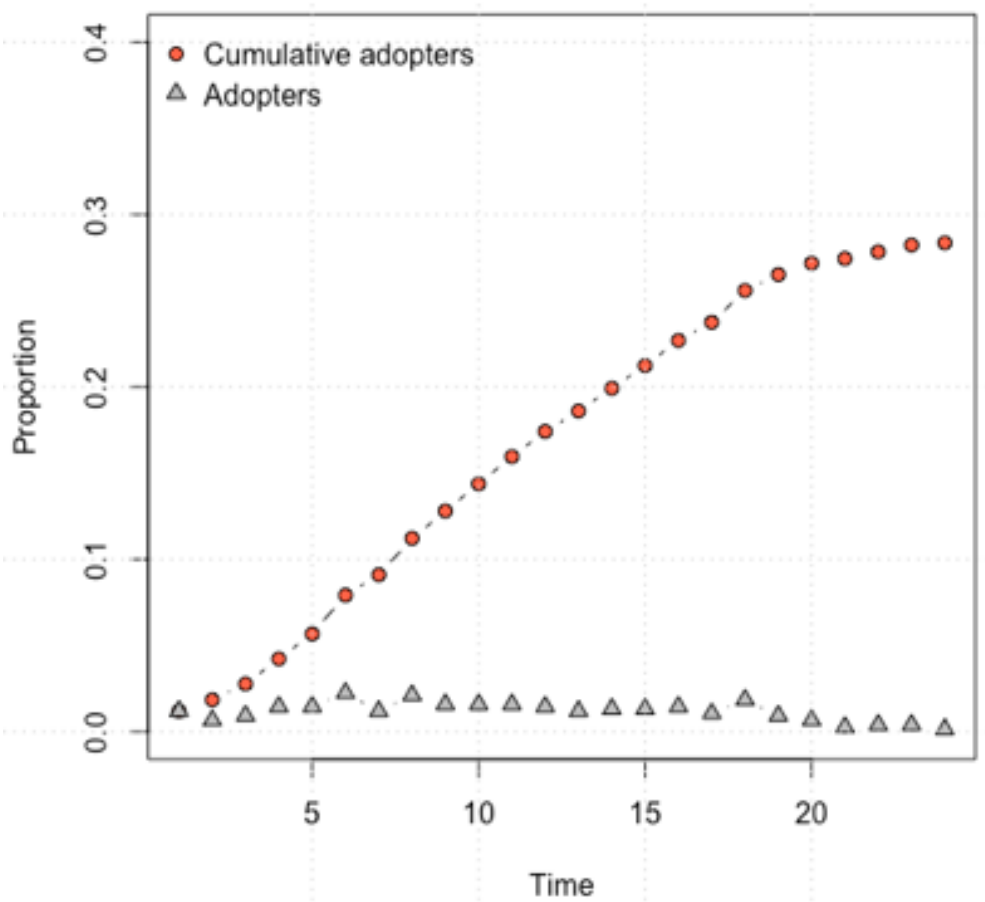

SI Figure 9 - Cumulative adopters over time for a single day in the dataset (31 ${ }^{\text {st }}$ March 2018) 
SI Table 2 - Example diffusion effects for a single day in the dataset (31 ${ }^{\text {st }}$ March 2018) across all 24 network slices

\begin{tabular}{|c|c|c|c|c|}
\hline Period & Adopters & Cumulative Adopters (\%) & Hazzard Rate & Moran's I (sd) \\
\hline 1 & 9 & $9(0.01)$ & - & $0.29(0.03) * * *$ \\
\hline 2 & 6 & $15(0.02)$ & 0.01 & $0.39(0.04) * * *$ \\
\hline 3 & 12 & $27(0.03)$ & 0.01 & $0.06(0.04) *$ \\
\hline 4 & 11 & $38(0.04)$ & 0.01 & $0.00(0.04)$ \\
\hline 5 & 13 & $51(0.06)$ & 0.02 & $0.04(0.04)$ \\
\hline 6 & 14 & $65(0.08)$ & 0.02 & $(0.04) * *$ \\
\hline 7 & 15 & $80(0.09)$ & 0.02 & $0.06(0.04)$ \\
\hline 8 & 20 & $100(0.12)$ & 0.03 & $0.06(0.04)$ \\
\hline 9 & 13 & $113(0.13)$ & 0.02 & $-0.05(0.04)$ \\
\hline 10 & 14 & $127(0.15)$ & 0.02 & $-0.07(0.04) *$ \\
\hline 11 & 16 & $143(0.17)$ & 0.02 & $-0.08(0.04) * *$ \\
\hline 12 & 21 & $164(0.19)$ & 0.03 & $-0.10(0.04) * * *$ \\
\hline 13 & 12 & $176(0.20)$ & 0.02 & $-0.12(0.04) * * *$ \\
\hline 14 & 6 & $182(0.21)$ & 0.01 & $-0.13(0.04) * * *$ \\
\hline 15 & 6 & $188(0.22)$ & 0.01 & $-0.13(0.04) * * *$ \\
\hline 16 & 10 & $198(0.23)$ & 0.01 & $-0.11(0.04) * * *$ \\
\hline 17 & 10 & $208(0.24)$ & 0.02 & $-0.11(0.04) * * *$ \\
\hline 18 & 6 & $214(0.25)$ & 0.01 & $-0.12(0.04) * * *$ \\
\hline 19 & 6 & $220(0.26)$ & 0.01 & $-0.13(0.04) * * *$ \\
\hline 20 & 2 & $222(0.26)$ & 0 & $-0.13(0.04) * * *$ \\
\hline 21 & 3 & $225(0.26)$ & 0 & $-0.14(0.04) * * *$ \\
\hline 22 & 2 & $227(0.26)$ & 0 & $-0.14(0.04) * * *$ \\
\hline 23 & 5 & $232(0.27)$ & 0.01 & $-0.12(0.04) * * *$ \\
\hline 24 & 4 & $236(0.27)$ & 0.01 & $-0.12(0.04) * * *$ \\
\hline
\end{tabular}

